\title{
Self-Concordant Barriers for Convex Approximations of Structured Convex Sets
}

\author{
Levent Tunçel* $\quad$ Arkadi Nemirovski ${ }^{\dagger}$
}

February 22, 2007 (revised: March 19, 2010)

\begin{abstract}
We show how to approximate the feasible region of structured convex optimization problems by a family of convex sets with explicitly given and efficient (if the accuracy of the approximation is moderate) self-concordant barriers. This approach extends the reach of the modern theory of interior-point methods, and lays the foundation for new ways to treat structured convex optimization problems with a very large number of constraints. Moreover, our approach provides a strong connection from the theory of self-concordant barriers to the combinatorial optimization literature on solving packing and covering problems.
\end{abstract}

Keywords: convex optimization, self-concordant barriers, semidefinite programming, interiorpoint methods, packing-covering problems

AMS Subject Classification: 90C25, 90C51, 90C22, 90C05, 52A41, 49M37, 90C59, 90C06

Communicated by Michael Todd.

This research was supported in part by Discovery Grants from NSERC.

\footnotetext{
*Corresponding Author: (ltuncel@math.uwaterloo.ca) Department of Combinatorics and Optimization, Faculty of Mathematics, University of Waterloo, Waterloo, Ontario, Canada.

${ }^{\dagger}$ (nemirovs@isye.gatech.edu) School of Industrial and Systems Engineering, Georgia Institute of Technology, Atlanta, Georgia, USA.
} 


\section{Introduction}

In modern convex optimization, when we consider polynomial-time algorithms, two families of algorithms stand out:

- interior-point methods,

- the ellipsoid method and related first-order methods.

Let $G \subset \mathbb{R}^{n}$ be a closed convex set with nonempty interior. Let $c \in \mathbb{R}^{n}$ be given. Then to solve the convex optimization problem

$$
\inf \{\langle c, x\rangle: x \in G\},
$$

modern interior-point methods usually require a computable self-concordant barrier function $f$ for $G$. Such functions (defined in the next section), completely describe the set $G$ and its boundary in a very special way. The ellipsoid method and related first-order methods require an efficient separation oracle for $G$. Such an oracle provides some local information about the set $G$ when it is called.

The ellipsoid method and first-order methods require very little global information about $G$ in each iteration. Another important difference between the two families of methods is that interior-point methods usually need $f^{\prime}, f^{\prime \prime}$ (the first and the second derivatives of the selfconcordant barrier $f$ ) at every iteration and the elementary operations needed can be as bad as $\Theta\left(n^{3}\right)$ per iteration. In contrast, the ellipsoid method and first-order methods can work with $O\left(n^{2}\right)$ operations per iteration and sometimes, for structured problems, they require much less than $\Theta\left(n^{2}\right)$ work per iteration.

If we have a self-concordant barrier for $G$ with barrier parameter $\vartheta$, then $O\left(\sqrt{\vartheta} \ln \left(\frac{1}{\epsilon}\right)\right)$ iterations of interior-point methods will suffice to produce an $\epsilon$-optimal solution (we assume that certain scale factors for the input problem are bounded by $O\left(\frac{1}{\epsilon}\right)$ ). This bound is significantly better than the best bounds of a similar nature for first-order algorithms. Moreover, whenever an application requires a very high accuracy of the solution, the practical performance of the interior-point algorithms is much better than the corresponding first-order methods.

Nevertheless, an important advantage of first-order methods can be observed at another extreme (low accuracy and extremely large dimension). First-order methods can be used to solve extremely large-scale problems (those for which performing even a single iteration of the interior-point methods is out of reach of the current hardware/software combinations) as long as the required accuracy of the final solution is modest, e.g., $10^{-2}$ or $10^{-4}$. Indeed, in many real applications, it does not even make sense to ask for more accuracy than $10^{-2}$, due to the nature of the problem and the data collected as well as the final (practical) uses of the approximate solutions.

Another important theoretical property of some first-order methods is that in some sense (in the black-box oracle model) for dimension-independent iteration bounds, they are optimal. That is, the upper bound on the number of iterations of a first-order method which only uses black-box subgradient information, $O\left(\frac{1}{\epsilon^{2}}\right)$ cannot be improved ([16]). However, as Nesterov [20] recently showed, the utilization of certain knowledge of the structure of the convex optimization problem at hand does help improve this upper bound very significantly to $O\left(\frac{1}{\epsilon}\right)$ (also see [19], [13]).

In combinatorial optimization, one of the most interesting and quite general structures is described by packing and covering problems; see the recent book by Cornuéjols [6]. Given an 
$m$-by- $n$ matrix $A$ with only 0,1 entries, and an objective function vector $c$, the combinatorial optimization problem

$$
\begin{aligned}
\max \langle c, x\rangle & \\
A x & \leq e \\
x & \in\{0,1\}^{n},
\end{aligned}
$$

(where $e$ is an all ones vector of appropriate size) is a packing problem. The combinatorial optimization problem

$$
\begin{aligned}
\min \langle c, x\rangle & \\
A x & \geq e \\
x & \in\{0,1\}^{n},
\end{aligned}
$$

is a covering problem. Both theoretical and practical approaches for solving the packing and the covering problems usually involve their linear programming relaxations, where $x \in\{0,1\}^{n}$ is replaced by $0 \leq x \leq e$. We will mostly deal with such problems and their generalizations.

Let $a_{i}$ denote the $i$ th row of $A$. Consider the function

$$
H(x):=\ln \left(\sum_{i=1}^{m} \exp \left\{\left\langle a_{i}, x\right\rangle\right\}\right) .
$$

This so-called exponential "potential" function $H(x)$ has been used in the context of approximately solving special classes of linear optimization problems (mainly those arising from covering and packing problems and minimum cost multi-commodity flow problems in combinatorial optimization), see $[24,10,11,22,7,4,5]$. In fact, such approaches proved useful and interesting even in the case of convex optimization problems with special convex functions and block diagonal structure in the constraints [9]. However, this function is not a self-concordant barrier and to the best of our knowledge, the role of self-concordant barriers in this context was previously non-existent.

Also, recently very good complexity bounds were obtained for the first-order algorithms for linear programming problems via approximations to the feasible regions which have certain symmetry properties [18].

In contrast to the existing work above, we will show how to approximate the feasible region of the structured optimization problem at hand by a family of convex sets for which we have efficient (if the accuracy of approximation is not too high) self-concordant barriers. We will construct self-concordant barriers with parameter $\vartheta$ for $\tilde{G}$ (a convex approximation of $G$ ) either independent of or only logarithmically dependent on the larger of the dimensions of the problem $(m)$, but strongly dependent on the goodness of our approximation to $G$.

Our work serves the following three purposes:

- We lay the foundation to bring the theory of modern interior-point methods (based on the self-concordance theory) closer to the ellipsoid method and more importantly to the recently proposed first-order methods in terms of the worst-case iteration bounds that are independent of the larger of the problem dimensions, but dependent on the desired accuracy $\epsilon$ as a polynomial in $\frac{1}{\epsilon}$.

- From a technical point of view, we show a new way of dealing with exponentially many terms in designing self-concordant barriers.

- We make a strong connection to the combinatorial optimization literature from the interiorpoint theory by providing new theoretical results for the packing-covering LPs (and their vast, nonpolyhedral generalizations) based on self-concordant barriers. 
A very important warning to the reader is about the computability of the barriers. Let $m$ be the larger of the dimensions in the problem data (and $n$ the smaller one). While the barrier parameters will grow at most with $\ln (m)$, if $m$ is very very large, say $m \approx 2^{n}$, then evaluating our barriers directly from the formulae we give can require $\Omega(m)\left(\approx 2^{n}\right)$ work. To avoid the resulting exponential complexity, one can resort to cutting-plane schemes based on these new barrier functions, or one can try to evaluate these large sums using efficient combinatorial techniques.

The paper is organized as follows: The next section deals with the LP relaxations of packing and covering problems. Two families of self-concordant barriers are derived, one based on the exponential function and the other based on the $p$-norm. Section 3 is very brief and simply points out that it is elementary to replace the nonnegative orthant by a closed convex cone. Section 4 generalizes the results much more significantly by replacing the linear functionals from Section 2 with linear operators and by replacing the linear inequalities of Section 2 with the partial orders induced by the cone of symmetric positive semidefinite matrices. In Section 5 , we illustrate that some basic patterns of the first three derivatives of the functions we use can be extended to a semi-infinite dimensional setting. The development up to Section 6 is not primal-dual symmetric. So, in Section 6 we study duality in this context and show how to generate a good dual feasible solution from a good, central primal solution. Finally, in Section 7 we conclude the technical results of the paper with a proposition showing that the square of the matrix $p$-norm function has Lipschitz continuous gradient with Lipschitz constant $2(p-1)$.

\section{Packing-Covering LPs}

In this section, we start our study with packing-covering LPs. First, we define the well-known notion of self-concordant barriers.

Definition 2.1 Let $G \subseteq \mathbb{R}^{n}$ be a closed convex set with nonempty interior. Then $f: \operatorname{int}(G) \rightarrow$ $\mathbb{R}$ is called a self-concordant barrier (s.c.b.) for $G$ with barrier parameter $\vartheta$ if the following conditions are satisfied:

- $f \in \mathcal{C}^{3}$, strictly convex on $\operatorname{int}(G)$;

- for every sequence $\left\{x^{(k)}\right\} \subset \operatorname{int}(G)$ such that $x^{(k)} \rightarrow \bar{x} \in \partial G, f\left(x^{(k)}\right) \rightarrow+\infty$;

- $\left|D^{3} f(x)[h, h, h]\right| \leq 2\left[D^{2} f(x)[h, h]\right]^{3 / 2}, \forall x \in \operatorname{int}(G), \forall h \in \mathbb{R}^{n}$;

- $(D f(x)[h])^{2} \leq \vartheta D^{2} f(x)[h, h]$, for every $x \in \operatorname{int}(G), h \in \mathbb{R}^{n}$.

Suppose $G$ is a closed convex cone with nonempty interior. Then a s.c.b. $f$ for $G$ with barrier parameter $\vartheta$ is called logarithmically homogeneous if

$$
f(t x)=f(x)-\vartheta \ln t, \quad \forall x \in \operatorname{int}(G), \forall t>0 .
$$

At this point, our first reaction to the packing/covering problems might be to consider the usual logarithmically homogeneous s.c. barriers for the underlying polyhedral cones. For the set $\left\{x \in \mathbb{R}_{+}^{n}: A x \leq e\right\}$, the s.c.b.

$$
f(x):=-\sum_{i=1}^{m} \ln \left(1-\left\langle a_{i}, x\right\rangle\right)-\sum_{j=1}^{n} \ln \left(x_{j}\right)
$$


has parameter value $(m+n)$. While this barrier has many very useful properties, its barrier parameter grows linearly with the number of constraints $m$. If $m \approx 2^{n}$ then the above approach with a direct application of the current theory of interior-point methods can only guarantee a complexity bound that is exponential in $n$. Next, as a second reaction to the situation, we might consider cutting-plane approaches based on the conventional s.c.b. given in (1). This second attempt, with a lot of work, can get around the exponential complexity bound; however, other, more subtle problems arise. For example, adding new inequalities on-the-fly increases the barrier parameter, while dropping constraints complicates the analysis of the algorithm.

We take a different approach... Note that

the constraints $A x \leq e$ are satisfied iff $\max _{i}\left\{\left\langle a_{i}, x\right\rangle\right\} \leq 1$.

Roughly stated, we consider two approximations to the latter condition:

1. Log-exp construction:

$$
\sum_{i=1}^{m} \exp \left\{L\left\langle a_{i}, x\right\rangle\right\} \leq \exp \{L\}, \text { for large } L
$$

2. $\|\cdot\|_{p}$-construction:

$$
\left(\sum_{i=1}^{m}\left\langle a_{i}, x\right\rangle^{p}\right)^{1 / p} \leq 1, \text { for large } p
$$

\subsection{The log-exp construction}

We begin with the results of approximating the constraint $\max _{i}\left\{\left\langle a_{i}, x\right\rangle\right\} \leq 1$ by $\sum_{i} \exp \left\{L\left\langle a_{i}, x\right\rangle\right\} \leq$ $\exp \{L\}$, for large $L$.

Proposition 2.1 Let $a_{i} \in \mathbb{R}_{+}^{n} \backslash\{0\}, i \in\{1,2, \ldots, m\}$, and let

$$
\begin{array}{rlr}
\tilde{G}(L):=\left\{x \in \mathbb{R}_{+}^{n}: \sum_{i=1}^{m} \exp \left\{L\left\langle a_{i}, x\right\rangle\right\} \leq \exp \{L\}\right\}, & {\left[L>\max \left[\ln m, \frac{3}{2}\right]\right] ;} \\
G(s):=\left\{x \in \mathbb{R}_{+}^{n}:\left\langle a_{i}, x\right\rangle \leq s, i \in\{1,2, \ldots, m\}\right\}, & {[s>0] .}
\end{array}
$$

Then

$$
G\left(1-\frac{\ln (m)}{L}\right) \subseteq \tilde{G}(L) \subseteq G(1)
$$

and the function

$$
\tilde{F}_{L}(x):=-\ln \left(L-\ln \left(\sum_{i} \exp \left\{L\left\langle a_{i}, x\right\rangle\right\}\right)\right)+\left(\frac{2 L}{3}\right)^{2} F_{-}(x),
$$

where

$$
F_{-}(x):=-\sum_{j=1}^{n} \ln \left(x_{j}\right)
$$

is a $\vartheta(L)$-self-concordant barrier for $\tilde{G}(L)$, with

$$
\vartheta(L):=1+\left(\frac{2 L}{3}\right)^{2} n .
$$


Proof. $1^{0}$. We clearly have

$$
\begin{aligned}
\sum_{i=1}^{m} \exp \left\{L\left\langle a_{i}, x\right\rangle\right\} \leq \exp \{L\} & \Rightarrow \exp \left\{L\left\langle a_{i}, x\right\rangle\right\} \leq \exp \{L\}, \quad \forall i \in\{1,2, \ldots, m\} \\
& \Rightarrow\left\langle a_{i}, x\right\rangle \leq 1, \quad \forall i \in\{1,2, \ldots, m\}
\end{aligned}
$$

whence $\tilde{G}(L) \subseteq G(1)$. On the other hand, if $x \in G(s), \sum_{i=1}^{m} \exp \left\{L\left\langle a_{i}, x\right\rangle\right\} \leq m \exp \{L s\}$, so that $s \leq 1-\frac{\ln (m)}{L} \Rightarrow x \in \tilde{G}(L)$. (2) is proved.

$2^{0}$. Let

$$
H(x):=\ln \left(\sum_{i} \exp \left\{\left\langle b_{i}, x\right\rangle\right\}\right), \text { where } b_{i}:=L a_{i}, \quad i \in\{1,2, \ldots, m\}
$$

Then

$$
\begin{array}{r}
D H(x)[h]=\frac{\sum_{i} \exp \left\{\left\langle b_{i}, x\right\rangle\right\}\left\langle b_{i}, h\right\rangle}{\sum_{i} \exp \left\{\left\langle b_{i}, x\right\rangle\right\}}=\sum_{i} p_{i}\left\langle b_{i}, h\right\rangle \\
{\left[p_{i}:=\frac{\exp \left\{\left\langle b_{i}, x\right\rangle\right\}}{\sum_{j} \exp \left\{\left\langle b_{j}, x\right\rangle\right\}}, \sum_{i} p_{i}=1\right]}
\end{array}
$$

whence

$$
\begin{aligned}
D^{2} H(x)[h, h] & \\
= & -\frac{\left(\sum_{i} \exp \left\{\left\langle b_{i}, x\right\rangle\right\}\left\langle b_{i}, h\right\rangle\right)^{2}}{\left(\sum_{i} \exp \left\{\left\langle b_{i}, x\right\rangle\right\}\right)^{2}}+\frac{\sum_{i} \exp \left\{\left\langle b_{i}, x\right\rangle\right\}\left\langle b_{i}, h\right\rangle^{2}}{\sum_{i} \exp \left\{\left\langle b_{i}, x\right\rangle\right\}}=-\left(\sum_{i} p_{i}\left\langle b_{i}, h\right\rangle\right)^{2}+\sum_{i} p_{i}\left\langle b_{i}, h\right\rangle^{2}=\sum_{i} p_{i} s_{i}^{2} \\
& {\left[s_{i}:=\left\langle b_{i}, h\right\rangle-\mu, \mu:=\sum_{j} p_{j}\left\langle b_{j}, h\right\rangle, \sum_{i} p_{i} s_{i}=0\right] }
\end{aligned}
$$

and finally

$$
\begin{aligned}
D^{3} H(x)[h, h, h] & \\
= & 2 \frac{\left(\sum_{i} \exp \left\{\left\langle b_{i}, x\right\rangle\right\}\left\langle b_{i}, h\right\rangle\right)^{3}}{\left(\sum_{i} \exp \left\{\left\langle b_{i}, x\right\rangle\right\}\right)^{3}}-3 \frac{\left(\sum_{i} \exp \left\{\left\langle b_{i}, x\right\rangle\right\}\left\langle b_{i}, h\right\rangle\right)\left(\sum_{i} \exp \left\{\left\langle b_{i}, x\right\rangle\right\}\left\langle b_{i}, h\right\rangle^{2}\right)}{\left(\sum_{i} \exp \left\{\left\langle b_{i}, x\right\rangle\right\}\right)^{2}}+\frac{\sum_{i} \exp \left\{\left\langle b_{i}, x\right\rangle\right\}\left\langle b_{i}, h\right\rangle^{3}}{\sum_{i} \exp \left\{\left\langle b_{i}, x\right\rangle\right\}} \\
= & 2\left(\sum_{i} p_{i}\left(s_{i}+\mu\right)\right)^{3}-3\left(\sum_{i} p_{i}\left(s_{i}+\mu\right)\right)_{i}\left(\sum_{i} p_{i}\left(s_{i}+\mu\right)^{2}\right)+\sum_{i} p_{i}\left(s_{i}+\mu\right)^{3} \\
= & 2 \mu^{3}-3 \mu\left(\mu^{2}+\sum_{i} p_{i} s_{i}^{2}\right)+\sum_{i} p_{i}\left(s_{i}^{3}+3 s_{i}^{2} \mu+3 s_{i} \mu^{2}+\mu^{3}\right) \\
= & 2 \mu^{3}-3 \mu^{3}-3 \mu \sum_{i} p_{i} s_{i}^{2}+\sum_{i} p_{i} s_{i}^{3}+3 \mu \sum_{i} p_{i} s_{i}^{2}+\mu^{3}=\sum_{i} p_{i} s_{i}^{3} .
\end{aligned}
$$

We arrive at the following observation called a compatibility result.

Lemma 2.1 . Let $x \in \tilde{G}(L) \bigcap \operatorname{int} \mathbb{R}_{+}^{n}$ and let $h$ be such that $x \pm h \in \mathbb{R}_{+}^{n}$. Then

$$
\left|D^{3} H(x)[h, h, h]\right| \leq 2 L D^{2} H(x)[h, h] .
$$


Proof. Indeed, if $x \pm h \in \mathbb{R}_{+}^{n}$, then $\left|h_{j}\right| \leq x_{j}, j \in\{1,2, \ldots, n\}$, whence $\left|\left\langle b_{i}, h\right\rangle\right| \leq\left\langle b_{i}, x\right\rangle \leq L$ (recall that $a_{i} \geq 0$ and $x \in \tilde{G}(L) \subseteq G(1)$ ). It follows that in the notation of $(5)-(7)$ we have $|\mu| \leq L$, whence $\left|s_{i}\right| \leq 2 L$. With this in mind, (8) follows from the concluding relations in (6) and (7).

For a cone $\mathbf{K} \subseteq \mathbb{R}^{N}$, and $u, v \in \mathbb{R}^{N}$, we write $u \leq_{\mathbf{K}} v$ to mean $(v-u) \in \mathbf{K}$. Now let us use the following result which originated from [21] (for the exact version below, see Theorem 9.1.1 in [14]):

\section{Lemma 2.2 Let}

- $G^{+} \subseteq \mathbb{R}^{N}$ be a closed convex domain, $F^{+}$be a $\vartheta_{+}$-self-concordant barrier for $G^{+}$and $\mathbf{K}$ be the recession cone of $G^{+}$;

- $G^{-}$be a closed convex domain in $\mathbb{R}^{n}$ and $F^{-}$be a $\vartheta_{-}$-self-concordant barrier for $G^{-}$;

- $\mathcal{A}: \operatorname{int} G^{-} \rightarrow \mathbb{R}^{N}$ be a $\mathcal{C}^{3}$ mapping such that $D^{2} \mathcal{A}(x)[h, h] \in-\mathbf{K}$ for all $x \in \operatorname{int} G^{-}$ and

$$
\forall\left(x \in \operatorname{int} G^{-}, \mathcal{A}(x) \in \operatorname{int} G^{+}\right) \forall\left(h, x \pm h \in G^{-}\right): D^{3} \mathcal{A}(x)[h, h, h] \leq_{\mathbf{K}}-3 \beta D^{2} \mathcal{A}(x)[h, h]
$$

- the set $G^{o}:=\left\{x \in \operatorname{int} G^{-}: \mathcal{A}(x) \in \operatorname{int} G^{+}\right\}$be nonempty.

Then $G^{o}$ is an open convex domain, and the function

$$
F^{+}(\mathcal{A}(x))+\max \left\{1, \beta^{2}\right\} F^{-}(x)
$$

is a self-concordant barrier for $\mathrm{cl} G^{o}$ with the parameter

$$
\vartheta:=\vartheta^{+}+\max \left\{1, \beta^{2}\right\} \vartheta_{-} .
$$

Note: In [21], relation (9) is assumed to be valid for all $x \in \operatorname{int} G^{-}$. However, the proof presented in [21] in fact requires only a weaker form of the assumption given by $(9)$; see [14].

Now let us specialize the data in the statement of Lemma 2.2 as follows:

- $G^{+}:=\{t \geq 0\} \subset \mathbb{R}, F^{+}(t):=-\ln (t)\left(\vartheta_{+}=1, \mathbf{K}:=\mathbb{R}_{+}\right)$

- $G_{-}:=\mathbb{R}_{+}^{n}, F^{-}(x):=-\sum_{j=1}^{n} \ln x_{j}\left(\vartheta_{-}=n\right)$;

- $\mathcal{A}(x):=L-\ln \left(\sum_{i=1}^{m} \exp \left\{L\left\langle a_{i}, x\right\rangle\right\}\right)$.

These data clearly satisfy all of the requirements from the premise of Lemma 2.2, except for (9); by Lemma 2.1, the latter requirement is also satisfied with $\beta=\frac{2 L}{3}$. Applying Lemma 2.2, we arrive at the desired result.

\subsection{The $\|\cdot\|_{p}$-construction}

Now, we consider approximating the constraint $\max _{i}\left\{\left\langle a_{i}, x\right\rangle\right\} \leq 1$ via a $p$-norm function. 
Proposition 2.2 Let $a_{i} \in \mathbb{R}_{+}^{n} \backslash\{0\}, i \in\{1,2, \ldots, m\}$. For $p \neq 0$ let

$$
H(x):=\left(\sum_{i=1}^{m}\left\langle a_{i}, x\right\rangle^{p}\right)^{1 / p}
$$

and let

$$
\mathbf{K}(p):= \begin{cases}\left\{(t, x) \in \mathbb{R} \times \mathbb{R}_{+}^{n}: t \geq H(x)\right\}, & p \geq 1 \\ \left\{(t, x) \in \mathbb{R} \times \mathbb{R}_{+}^{n}: t \leq H(x)\right\}, & p \leq 1, p \neq 0 .\end{cases}
$$

Then

$$
\begin{aligned}
& p \geq 1 \Rightarrow\left\{(t, x) \in \mathbb{R} \times \mathbb{R}_{+}^{n}: \max _{i}\left\langle a_{i}, x\right\rangle \leq \frac{t}{m^{1 / p}}\right\} \subseteq \mathbf{K}(p) \subseteq\left\{(t, x) \in \mathbb{R} \times \mathbb{R}_{+}^{n}: \max _{i}\left\langle a_{i}, x\right\rangle \leq t\right\}, \\
& p<0 \Rightarrow\left\{(t, x) \in \mathbb{R} \times \mathbb{R}_{+}^{n}: \min _{i}\left\langle a_{i}, x\right\rangle \geq \frac{t}{m^{1 / p}}\right\} \subseteq \mathbf{K}(p) \subseteq\left\{(t, x) \in \mathbb{R} \times \mathbb{R}_{+}^{n}: \min _{i}\left\langle a_{i}, x\right\rangle \geq t\right\}
\end{aligned}
$$

and the function

$$
F_{p}(x):=\left(\frac{2|p-2|+3}{3}\right)^{2} F_{-}(x)-\left\{\begin{array}{ll}
\ln (t-H(x)), & p \geq 1 \\
\ln (H(x)-t), & p \leq 1, p \neq 0
\end{array}, F_{-}(x):=-\sum_{j=1}^{n} \ln \left(x_{j}\right)\right.
$$

is a $\vartheta_{p}$-logarithmically homogeneous self-concordant barrier for $\mathbf{K}(p)$, with

$$
\vartheta_{p}:=1+\left(\frac{2|p-2|+3}{3}\right)^{2} n .
$$

Proof We proceed as in the proof of Proposition 2.1. (10) follows straightforwardly, and then as in the latter proof, the only facts to be verified are that

(a) $H$ is convex on int $\mathbb{R}_{+}^{n}$ when $p \geq 1$ and is concave on $\operatorname{int} \mathbb{R}_{+}^{n}$ when $p \leq 1, p \neq 0$;

(b) whenever $x \in \operatorname{int} \mathbb{R}_{+}^{n}$ and $h$ is such that $x \pm h \in \mathbb{R}_{+}^{n}$, we have

$$
\left|D^{3} H(x)[h, h, h]\right| \leq(2|p-2|+3)\left|D^{2} H(x)[h, h]\right| .
$$

Assume that $p \neq 0$. Given $x \in \operatorname{int} \mathbb{R}_{+}^{n}, a_{i} \in \mathbb{R}_{+}^{n} \backslash\{0\}, h \in \mathbb{R}^{n}$ such that $x \pm h \in \mathbb{R}_{+}^{n}$, let us set

$$
p_{i}(x):=\frac{\left\langle a_{i}, x\right\rangle^{p}}{\sum_{j}\left\langle a_{j}, x\right\rangle^{p}}, \delta_{i}(x):=\frac{\left\langle a_{i}, h\right\rangle}{\left\langle a_{i}, x\right\rangle}, \mu(x):=\sum_{i} p_{i}(x) \delta_{i}(x), s_{i}(x):=\delta_{i}(x)-\mu(x) .
$$

We have

$$
\begin{aligned}
& \qquad H(x)[h]=H(x) \mu(x) \\
& D^{2} H(x)[h, h]=H(x)\left[\mu^{2}(x)+D \mu(x)[h]\right] \\
& =H(x)\left[\mu^{2}(x)+\left[\sum_{i}(p-1) p_{i}(x) \delta_{i}^{2}(x)-p \mu^{2}(x)\right]\right] \\
& =H(x)\left[\mu^{2}(x)+(p-1) \sum_{i} p_{i}(x) s_{i}^{2}(x)+(p-1) \mu^{2}(x)-p \mu^{2}(x)\right] \\
& =(p-1) H(x) \sum_{i} p_{i}(x) s_{i}^{2}(x) ;
\end{aligned}
$$




$$
\begin{aligned}
& D^{3} H(x)[h, h, h]=H(x)\left[\mu^{3}(x)+\mu(x) D \mu(x)[h]+2 \mu(x) D \mu(x)[h]+D^{2} \mu(x)[h, h]\right] \\
& =H(x)\left[\mu^{3}(x)+3 \mu(x) D \mu(x)[h]+D^{2} \mu(x)[h, h]\right] \\
& =H(x)\left[\mu^{3}(x)+3 \mu(x) D \mu(x)[h]-2 p \mu(x) D \mu(x)[h]\right. \\
& \left.-2(p-1) \sum_{i} p_{i}(x) \delta_{i}^{3}(x)+p(p-1) \sum_{i} p_{i}(x) \delta_{i}^{3}(x)-p(p-1)\left(\sum_{i} p_{i}(x) \delta_{i}^{2}\right) \mu(x)\right] \\
& =H(x)\left[\mu^{3}(x)+(3-2 p) \mu(x)\left[(p-1) \sum_{i} p_{i}(x) \delta_{i}^{2}(x)-p \mu^{2}(x)\right]\right. \\
& \left.+(p-1)(p-2) \sum_{i} p_{i}(x) \delta_{i}^{3}(x)-p(p-1) \mu(x) \sum_{i} p_{i}(x) \delta_{i}^{2}(x)\right] \\
& \quad=H(x)\left[[1-p(3-2 p)] \mu^{3}(x)+(p-1)(3-3 p) \mu(x)\left[\sum_{i} p_{i}(x) s_{i}^{2}(x)+\mu^{2}(x)\right]\right. \\
& \left.\quad+(p-1)(p-2) \sum_{i} p_{i}(x)\left[s_{i}^{3}(x)+3 s_{i}^{2}(x) \mu(x)+3 s_{i}(x) \mu^{2}(x)+\mu^{3}(x)\right]\right] \\
& \quad=H(x)\left[(p-1)(p-2) \sum_{i} p_{i}(x) s_{i}^{3}(x)-3(p-1) \mu(x) \sum_{i} p_{i}(x) s_{i}^{2}(x)\right] .
\end{aligned}
$$

Since $a_{i} \in \mathbb{R}_{+}^{n}, x \in \operatorname{int} \mathbb{R}_{+}^{n}$ and $x \pm h \in \mathbb{R}_{+}^{n}$, we have $\left|\delta_{i}(x)\right| \leq 1$, whence $|\mu(x)| \leq 1$ and $\left|s_{i}(x)\right| \leq 2$. We see that $H$ is convex when $p \geq 1, H$ is concave when $p \leq 1, p \neq 0$ and (13) holds.

Corollary 2.1 Let $a_{i} \in \mathbb{R}_{+}^{n} \backslash\{0\}$ and $\pi_{i}>0$, for every $i \in\{1,2, \ldots, m\}$ be such that $\sum_{i=1}^{m} \pi_{i}=1$. Then the concave function $f(t, x)=\left\langle a_{1}, x\right\rangle^{\pi_{1}}\left\langle a_{2}, x\right\rangle^{\pi_{2}} \cdots\left\langle a_{m}, x\right\rangle^{\pi_{m}}-t$ considered as a mapping from int $G_{-}:=\mathbb{R} \times \operatorname{int} \mathbb{R}_{+}^{n}$ to $\mathbb{R}$, satisfies (9) with $G_{+}:=\mathbb{R}_{+}$and $\beta=\frac{7}{3}$ :

$$
x>0, x \pm h \geq 0 \Rightarrow\left|D_{x}^{3} f(t, x)[h, h, h]\right| \leq-7 D_{x}^{2} f(t, x)[h, h] .
$$

Proof. Indeed, we have seen that if $0<p<1$ then $H_{p}(x)=\left(\sum_{i=1}^{m}\left\langle a_{i}, x\right\rangle^{p}\right)^{1 / p}$ satisfies the relation

$$
x>0, x \pm h \geq 0 \Rightarrow\left|D^{3} H_{p}(x)[h, h, h]\right| \leq-(2|p-2|+3) D^{2} H_{p}(x)[h, h] .
$$

It remains to note that as $p \rightarrow 0^{+}$, the functions $\frac{H_{p}(x)}{m^{1 / p}}$ converge uniformly along with derivatives on every compact subset of int $\mathbb{R}_{+}^{n}$ to the function $g(x)=\left(\prod_{i=1}^{m}\left\langle a_{i}, x\right\rangle\right)^{1 / m}$. It follows that the desired inequality is valid when $\pi_{1}=\pi_{2}=\cdots=\pi_{m}=\frac{1}{m}$. This fact in turn implies that the desired relation is valid when all $\pi_{i}$ are rational, which in turn implies the validity of the statement for all $\pi_{i}>0$ with the unit sum.

From now on, all $O(1)$ 's are positive absolute constants. As a direct consequence of the last corollary, we also have the following fact.

Corollary 2.2 The function

$$
F(x):=-\ln \left(\prod_{i=1}^{m}\left\langle a_{i}, x\right\rangle^{\pi_{i}}-t\right)-\left(\frac{7}{3}\right)^{2} \sum_{j=1}^{n} \ln x_{j}
$$

is an $O(1) n$-self-concordant barrier for the convex set

$$
\left\{(t, x) \in \mathbb{R} \times \mathbb{R}_{+}^{n}: \prod_{i=1}^{m}\left\langle a_{i}, x\right\rangle^{\pi_{i}} \geq t\right\} .
$$




\subsection{Required accuracy and the barrier parameter}

In many problems from combinatorial optimization, we are usually interested in computing the maximum (or minimum) cardinality sets satisfying a certain criterion, for example, maximum cardinality stable set, maximum cardinality matching, minimum cardinality node cover, minimum number of colors needed to color a graph. In these cases, a $\frac{1}{n}$-approximation to the underlying polytope usually yields an exact algorithm to compute that maximum or minimum value. First, we work with the desired tolerance $\epsilon$, then we will substitute $\epsilon=\frac{1}{n}$. Thus, in our notation above, for the log-exp construction, we need

$$
1-\frac{\ln (m)}{L} \geq 1-\epsilon \text { which is equivalent to } L \geq \frac{\ln (m)}{\epsilon} .
$$

Therefore, the self-concordance parameter of $\tilde{F}_{L}$ is $1+\frac{4 n \ln ^{2}(m)}{9 \epsilon^{2}}$ and in the case of $\epsilon=1 / n$ and $m=O\left(2^{n}\right)$, we arrive at $\vartheta(L)=O\left(n^{5}\right)$. This would imply an iteration bound of $O\left(n^{2.5} \ln (n)\right)$.

Let us do the analogous computation for the $\|\cdot\|_{p}$-construction. For packing problems $(p \geq 1)$, we need $\frac{1}{m^{1 / p}} \geq 1-\epsilon$, which is equivalent to $p \geq \frac{\ln (m)}{\ln \left(\frac{1}{1-\epsilon}\right)}$. For covering problems $(p<0)$, we need $|p| \geq \frac{\ln (m)}{\ln \left(\frac{1}{1-\epsilon}\right)}$. In either case (packing or covering), the complexity bound is dictated by

$$
\vartheta_{p}=O\left[\frac{n \ln ^{2}(m)}{\ln ^{2}\left(\frac{1}{1-\epsilon}\right)}\right]
$$

If $m=2^{n}$ and $\epsilon=1 / n$, we have $\vartheta_{p}=O\left(n^{5}\right)$ which leads to the iteration bound $O\left(n^{2.5} \ln (n)\right)$.

One distinct advantage of these new self-concordant barriers is that their barrier parameter can be kept fixed as we add cutting-planes. Let us fix the desired tolerance $\epsilon$. In many cuttingplane schemes that admit polynomial or pseudo-polynomial complexity analyses, one can bound from above the number of cutting-planes that will be generated by the cutting-plane scheme. Let us denote this upper bound by $\bar{m}$ and suppose that $\bar{m}$ is bounded by a polynomial function of the input. When we construct our barrier $\tilde{F}_{L}$ (or $F_{p}$ ), we can compute $L$ (or $p$ ) using the upper bound $\bar{m}$. Then in a cutting-plane scheme, as we add new constraints, the barrier parameter stays fixed at $\vartheta(L)$ (or $\vartheta_{p}$ ). This can be a significant advantage at least in theoretical work on such algorithms. In many cutting-plane schemes, $\bar{m}$ is proven to be $O\left(n^{4}\right)$. In such a situation, when $\bar{m}$ is bounded by a polynomial function of $n$, we obtain

$$
\vartheta(L)=O\left(n^{3} \ln ^{2}(n)\right) \text { for the log-exp construction, }
$$

and

$$
\vartheta_{p}=O\left(n^{3} \ln ^{2}(n)\right) \text { for the }\|\cdot\|_{p^{-} \text {-construction. }}
$$

These lead to the iteration bound: $O\left(n^{1.5} \ln ^{2}(n)\right)$ for both constructions. Note that our barriers and their first and second derivatives can be evaluated in polynomial time in these situations.

In what follows, we call $\mathbb{R}_{+}^{n}$ the primary domain, and name the set

$$
\left\{x \in \mathbb{R}^{n}:\left\langle a_{i}, x\right\rangle \leq 1, i \in\{1,2, \ldots, m\}\right\}
$$

the secondary domain. Next, we show that the above approach can be widely generalized in terms of each of these domains. 


\section{Generalization of the Primary Domain}

From the outlined proofs it is clear that the above results remain valid when

- the primary domain (nonnegative orthant $\mathbb{R}_{+}^{n}$ ) is replaced by an arbitrary closed convex pointed cone $\mathbf{K}$ with nonempty interior,

- assumption $a_{i} \in \mathbb{R}_{+}^{n} \backslash\{0\}$ is replaced by $a_{i} \in \mathbf{K}^{*} \backslash\{0\}$, where $\mathbf{K}^{*}$ is the cone dual to $\mathbf{K}$,

$$
\mathbf{K}^{*}:=\left\{s \in \mathbb{R}^{n}:\langle x, s\rangle \geq 0, \quad \forall x \in \mathbf{K}\right\}
$$

- the barrier $F_{-}(x)=-\sum_{j=1}^{n} \ln \left(x_{j}\right)$ for $\mathbb{R}_{+}^{n}$ is replaced by a $\vartheta_{-}$-self-concordant barrier $F$ for $\mathbf{K}$, and the factor $n$ in (4), (12) is replaced by $\vartheta_{-}$.

\section{Generalization of the Secondary Domain}

In addition to the above generalization of the primary domain, we can also generalize each constraint of the secondary domain. For example, for each $i$, we can replace $\left\langle a_{i}, x\right\rangle \leq 1$ by $\mathcal{A}_{i}(x) \preceq I$, where $\mathcal{A}_{i}: \mathbb{R}^{n} \rightarrow \mathbb{S}^{\ell_{i}}$ a linear map (from $\mathbb{R}^{n}$ to the space of $\ell_{i}$-by- $\ell_{i}$ symmetric matrices with real entries) and " $\preceq$ " is the partial order induced by the cone of positive semidefinite matrices, $\mathbb{S}_{+}^{\ell_{i}}$, in $\mathbb{S}^{\ell_{i}}$. So, $\mathcal{A}_{i}(x) \preceq I$ means $\left[I-\mathcal{A}_{i}(x)\right] \in \mathbb{S}_{+}^{\ell_{i}}$. The results of Sections 2 and 3 are included as the special case $\ell_{i}=1$ for every $i \in\{1,2, \ldots, m\}$. We first present the generalization of the $p$-norm construction.

\subsection{The $\|\cdot\|_{p}$-construction}

\subsubsection{Compatibility}

Let $\operatorname{Tr}: \mathbb{S}^{d} \rightarrow \mathbb{R}$ denote the trace and $\mathbb{S}_{++}^{d}$ denote the interior of $\mathbb{S}_{+}^{d}$. We sometimes write $y \succ 0$ to mean that $y$ is a symmetric positive definite matrix. We start by establishing the following fact (whose proof is in the appendix, as Proposition A.1):

Proposition 4.1 Let $p,|p| \geq 2$, be integer. Consider the following functions of $y \in \mathbb{S}_{++}^{d}$ :

$$
F(y):=\operatorname{Tr}\left(y^{p}\right), f(y):=(F(y))^{1 / p} .
$$

Then $f$ is convex when $p \geq 2, f$ is concave when $p \leq-2$, and

$$
y \succ 0, y \pm h \succeq 0 \Rightarrow\left|D^{3} f(y)[h, h, h]\right| \leq O(1)|p|\left|D^{2} f(y)[h, h]\right| .
$$

The following statement is the matrix analogue of Corollary 2.1.

Proposition 4.2 Let $f(y)=\operatorname{Det}^{1 / m}(y)$, where $y \in \mathbb{S}_{++}^{m}$. Then $f$ is concave on $\mathbb{S}_{++}^{m}$ and

$$
y \succ 0, y \pm h \succeq 0 \Rightarrow\left|D^{3} f(y)[h, h, h]\right| \leq-7 D^{2} f(y)[h, h] .
$$


Proof. Setting $H(y)=\ln \operatorname{Det}(y), y \succ 0$, we have

$$
\begin{aligned}
f(y)= & \exp \{H(y) / m\} \\
D f(y)[h]= & f(y)\left(m^{-1} D H(y)[h]\right)=f(y)\left(m^{-1} \operatorname{Tr}\left(y^{-1} h\right)\right), \\
D^{2} f(y)[h, h]= & f(y)\left(m^{-1} D H(y)[h]\right)^{2}+f(y)\left(m^{-1} D^{2} H(y)[h, h]\right) \\
= & f(y)\left[\left(m^{-1} \operatorname{Tr}\left(y^{-1} h\right)\right)^{2}-m^{-1} \operatorname{Tr}\left(y^{-1} h y^{-1} h\right)\right] \\
D^{3} f(y)[h, h, h]= & f(y)\left(m^{-1} D H(y)[h]\right)\left[\left(m^{-1} \operatorname{Tr}\left(y^{-1} h\right)\right)^{2}-m^{-1} \operatorname{Tr}\left(y^{-1} h y^{-1} h\right)\right] \\
& +f(y)\left[-2\left(m^{-1} \operatorname{Tr}\left(y^{-1} h\right)\right)\left(m^{-1} \operatorname{Tr}\left(y^{-1} h y^{-1} h\right)\right)+2 m^{-1} \operatorname{Tr}\left(y^{-1} h y^{-1} h y^{-1} h\right)\right]
\end{aligned}
$$

Setting $\eta=y^{-1 / 2} h y^{-1 / 2}$ and denoting by $\lambda(u)$ the vector of eigenvalues of $u \in \mathbb{S}^{n}$, by $\mathbf{E}\{g\}$ the average of the coordinates of a vector $g$, and by $[g]^{k}, g$ being a vector, the vector with coordinates $g_{i}^{k}$, we get

$$
\begin{aligned}
D f(y)[h]= & f(y) \mathbf{E}\{\lambda(\eta)\}=f(y) \mu \\
& {[\mu:=\mathbf{E}\{\lambda(\eta)\}] } \\
D^{2} f(y)[h, h]= & f(y)\left[\mu^{2}-\mathbf{E}\left\{[\lambda(\eta)]^{2}\right\}\right]=f(y) \mathbf{E}\left\{[\sigma]^{2}\right\} \\
& {\left[\sigma_{i}:=\lambda_{i}(\eta)-\mu\right] } \\
D^{3} f(y)[h, h, h]= & f(y)\left[\mu^{3}-3 \mu \mathbf{E}\left\{[\lambda(\eta)]^{2}\right\}+2 \mathbf{E}\left\{[\lambda(\eta)]^{3}\right\}\right] \\
= & f(y)\left[\mu^{3}-3 \mu\left[\mu^{2}+\mathbf{E}\left\{[\sigma]^{2}\right\}\right]+2 \mathbf{E}\left\{\mu^{3} e+3 \mu^{2} \sigma+3 \mu[\sigma]^{2}+[\sigma]^{3}\right\}\right] \\
= & f(y)\left[3 \mu \mathbf{E}\left\{[\sigma]^{2}\right\}+2 \mathbf{E}\left\{[\sigma]^{3}\right\}\right] .
\end{aligned}
$$

Under the premise in (15), we have $\|\lambda(\eta)\|_{\infty} \leq 1$, whence $|\mu| \leq 1$ and $\|\sigma\|_{\infty} \leq 2$, which, in view of the above formulas for the derivatives of $f$, immediately implies the conclusion in (15).

\subsubsection{The $\|\cdot\|_{p}$-barrier}

Now, we are ready to state and prove the main result for the matrix generalization of the $p$-norm construction.

Theorem 4.1 Let $\mathbf{K}$ be a closed convex cone with a nonempty interior in $\mathbb{R}^{n}, F_{-}(x)$ be a $\vartheta_{-}$self-concordant barrier for $\mathbf{K}$, and let $\mathcal{A}_{i}: \mathbb{R}^{n} \rightarrow \mathbb{S}^{\ell_{i}}, i \in\{1,2, \ldots, m\}$, be linear mappings such that

$$
x \in \operatorname{int} \mathbf{K} \Rightarrow \mathcal{A}_{i}(x) \succ 0, i \in\{1,2, \ldots, m\} .
$$

Let us set

$$
\mathcal{A}(x):=\operatorname{Diag}\left\{\mathcal{A}_{1}(x), \mathcal{A}_{2}(x), \ldots, \mathcal{A}_{m}(x)\right\} .
$$

(i) Given integer $p \geq 2$, consider the function

$$
g(x):=\left(\operatorname{Tr}\left([\mathcal{A}(x)]^{p}\right)\right)^{1 / p}: \operatorname{int} \mathbf{K} \rightarrow \mathbb{R} .
$$

This function is convex and $O(1) p$-compatible with its domain:

$$
x \in \operatorname{int} \mathbf{K}, x \pm h \in \mathbf{K} \Rightarrow\left|D^{3} g(x)[h, h, h]\right| \leq O(1) p D^{2} g(x)[h, h],
$$

so that the function

$$
\Phi(t, x)=-\ln (t-g(x))+O(1) p^{2} F_{-}(x)
$$

is a self-concordant barrier with the parameter

$$
\vartheta=1+O(1) p^{2} \vartheta_{-}
$$


for the cone

$$
\mathbf{K}(p)=\left\{(t, x) \in \mathbb{R}_{+} \times \mathbf{K}: g(x) \leq t\right\} .
$$

Moreover, with

$$
\theta=\left(\sum_{i=1}^{m} \ell_{i}\right)^{1 / p}
$$

we have

$$
\mathbf{M}_{\theta} \subseteq \mathbf{K}(p) \subseteq \mathbf{M}_{1}
$$

where $\mathbf{M}_{r}$ is the cone

$$
\left\{(t, x): x \in \mathbf{K}, \mathcal{A}_{i}(x) \preceq \frac{t}{r} I, i \in\{1,2, \ldots, m\}\right\} .
$$

(ii) Given integer $p \leq-2$, consider the function

$$
g(x):=\left(\operatorname{Tr}\left([\mathcal{A}(x)]^{p}\right)\right)^{1 / p}: \operatorname{int} \mathbf{K} \rightarrow \mathbb{R} .
$$

This function is concave and $O(1)|p|$-compatible with its domain:

$$
x \in \operatorname{int} \mathbf{K}, x \pm h \in \mathbf{K} \Rightarrow\left|D^{3} g(x)[h, h, h]\right| \leq-O(1)|p| D^{2} g(x)[h, h],
$$

so that the function

$$
\Phi(t, x)=-\ln (g(x)-t)+O(1) p^{2} F_{-}(x)
$$

is a self-concordant barrier with the parameter

$$
\vartheta=1+O(1) p^{2} \vartheta_{-}
$$

for the cone

$$
\mathbf{K}(p)=\{(t, x) \in \mathbb{R} \times \mathbf{K}: g(x) \geq t\} .
$$

Moreover, with

$$
\theta=\left(\sum_{i=1}^{m} \ell_{i}\right)^{1 / p}
$$

we have

$$
\mathbf{N}_{\theta} \subseteq \mathbf{K}(p) \subseteq \mathbf{N}_{1}
$$

where $\mathbf{N}_{r}$ is the cone

$$
\left\{(t, x): x \in \mathbf{K}, \mathcal{A}_{i}(x) \succeq \frac{t}{r} I, i \in\{1,2, \ldots, m\}\right\} .
$$

Proof. All we need is to prove (16) and (18); the statements on self-concordance of $\Phi(\cdot)$ are direct consequences of the former relations (see [21]), and the inclusions (17), (19) are evident.

To prove (16) (the proof of (18) is similar), let $N:=\sum_{i} \ell_{i}$ and let $f(y):=\left(\operatorname{Tr}\left(y^{p}\right)\right)^{1 / p}$ : $\mathbb{S}_{++}^{N} \rightarrow \mathbb{R}$. Assuming that $x, h$ satisfy the premise in $(16)$, let us set $y:=\mathcal{A}(x), \hat{h}:=\mathcal{A}(h)$. Since $\mathcal{A}(\cdot)$ is a linear mapping which maps int $\mathbf{K}$ into $\mathbb{S}_{++}^{N}$, we have

$$
y \succ 0, \quad y \pm \hat{h} \succeq 0,
$$

whence, by Proposition 4.1,

$$
\left|D^{3} f(y)[\hat{h}, \hat{h}, \hat{h}]\right| \leq O(1) p D^{2} f(y)[\hat{h}, \hat{h}] .
$$

Since $D^{\kappa} g(x)[h, \ldots, h]=D^{\kappa} f(y)[\hat{h}, \ldots, \hat{h}]$, we see that the conclusion in (16) indeed is true. 


\subsection{The log-exp construction}

Our log-exp construction for packing and covering LPs also generalize to the matrix case. In what follows, we use the matrix exponential. That is, for $x \in \mathbb{S}^{n}$,

$$
\exp \{x\}:=\sum_{k=0}^{\infty} \frac{1}{k !} x^{k} .
$$

We first begin with the compatibility result (whose proof is in the appendix, as Proposition A.2).

Proposition 4.3 Let

$$
F(y):=\operatorname{Tr}(\exp \{y\}), f(y):=\ln (F(y)) \quad\left[y \in \mathbb{S}^{d}\right]
$$

Then

$$
(L I \succeq y \succ 0, y \pm h \succeq 0) \Rightarrow\left|D^{3} f(y)[h, h, h]\right| \leq O(1) L D^{2} f(y)[h, h] .
$$

\subsubsection{The log-exp barrier}

As before, now that the compatibility result is established, we can state and prove the main theorem for the log-exp construction.

Theorem 4.2 Let $\mathbf{K}$ be a closed convex cone with a nonempty interior in $\mathbb{R}^{n}, F_{-}(x)$ be a $\vartheta_{-}$self-concordant barrier for $\mathbf{K}$, and let $\mathcal{A}_{i}: \mathbb{R}^{n} \rightarrow \mathbb{S}^{\ell_{i}}, i \in\{1,2, \ldots, m\}$, be linear mappings such that

$$
x \in \operatorname{int} \mathbf{K} \Rightarrow \mathcal{A}_{i}(x) \succ 0, i \in\{1,2, \ldots, m\}
$$

Let us set

$$
\mathcal{A}(x):=\operatorname{Diag}\left\{\mathcal{A}_{1}(x), \mathcal{A}_{2}(x), \ldots, \mathcal{A}_{m}(x)\right\} .
$$

Given $L>\ln (N)$, where $N:=\sum_{i=1}^{m} \ell_{i}$, consider the function

$$
g(x):=\ln (\operatorname{Tr}(\exp \{L \mathcal{A}(x)\})): \operatorname{int} \mathbf{K} \rightarrow \mathbb{R} .
$$

This function is convex and satisfies the relation:

$$
x \in \operatorname{int} \mathbf{K}, x \pm h \in \mathbf{K}, g(x) \leq L \Rightarrow\left|D^{3} g(x)[h, h, h]\right| \leq O(1) L D^{2} g(x)[h, h] .
$$

Consequently, the function

$$
\Phi(x)=-\ln (L-g(x))+O(1) L^{2} F_{-}(x)
$$

is a self-concordant barrier with the parameter

$$
\vartheta=1+O(1) L^{2} \vartheta_{-}
$$

for the set

$$
\mathbf{K}(L):=\operatorname{cl}\{x \in \operatorname{int} \mathbf{K}: g(x) \leq L\} .
$$

Moreover, when $\delta \in(0,1)$ and $L \geq \frac{\ln (N)}{\delta}$, we have

$\left\{x \in \mathbf{K}: \mathcal{A}_{i}(x) \preceq(1-\delta) I, i \in\{1,2, \ldots, m\}\right\} \subseteq \mathbf{K}(L) \subseteq\left\{x \in \mathbf{K}: \mathcal{A}_{i}(x) \preceq I, i \in\{1,2, \ldots, m\}\right\}$. 
Proof. As in the proof of Proposition 2.1, all we need to do is to verify (22), which is immediate. Indeed, let $x, h$ satisfy the premise in $(22)$, and let $y=L \mathcal{A}(x), \hat{h}=L \mathcal{A}(h)$. Since $\mathcal{A}$ is a linear mapping which maps int $\mathbf{K}$ into int $\mathbb{S}_{+}^{N}$, we have $y \succ 0$ and $y \pm \hat{h} \succeq 0$. Moreover, from $g(x) \leq L$ it follows that $y \preceq L I$. Setting $f(y)=\ln \operatorname{Tr}(\exp \{y\})$, we have $g(x)=f(L \mathcal{A}(x))$, whence $D^{k} g(x)[h, \ldots, h]=D^{k} f(y)[\hat{h}, \ldots, \hat{h}]$, for all $k \in \mathbb{Z}_{+}$. As we have seen, $y, \hat{h}$ satisfy the premise in (21); thus, applying Proposition 4.3, we arrive at the conclusion in (22).

\section{A Generalization to Convex Semi-infinite Programming}

The reader must have recognized that there are certain uniform structures to the derivatives of the functions which we utilized in this paper. These structures seem critical in securing the necessary inequalities in the barrier calculus of Nesterov and Nemirovski [21] and in turn obtaining the related barriers with the desired self-concordance properties. In this section, we show that many of these properties generalize to the case when our variable is infinite dimensional.

Let $f_{\alpha}(x)>0$ for all $x \in \mathbb{R}_{++}^{n}$ and $\mu$ be a measure on the set of indices $T$. Let us define

$$
\Phi(x):=\left(\int_{T} f_{\alpha}^{p}(x) \mu(d \alpha)\right)^{1 / p} .
$$

Then for all $0 \neq p \in \mathbb{R}$, we have

$$
\begin{aligned}
& D \Phi(x)[h]=\Phi(x) \mathbf{E}_{x}\left\{S_{x, h}(\cdot)\right\}, \\
& \text { where } S_{x, h}(\alpha):=\frac{D f_{\alpha}(x)[h]}{f_{\alpha}(x)}, \pi_{x}(\alpha):=\frac{f_{\alpha}^{p}(x)}{\int f_{\beta}^{p}(x) \mu(d \beta)} \text { and } \mathbf{E}_{x}\{g(\cdot)\}=\int g(\alpha) \pi_{x}(\alpha) \mu(d \alpha) ; \\
& D^{2} \Phi(x)[h, h]=\Phi(x)\left[(p-1) \mathbf{E}_{x}\left\{\sigma_{x, h}^{2}\right\}+\mathbf{E}_{x}\left\{\frac{D^{2} f_{\alpha}(x)[h, h]}{f_{\alpha}(x)}\right\}\right], \\
& \text { where } \sigma_{x, h}(\alpha):=S_{x, h}(\alpha)-\mathbf{E}_{x}\left\{S_{x, h}(\cdot)\right\} \\
& D^{3} \Phi(x)[h, h, h]=\Phi(x)\left[\mathbf{E}_{x}\left\{\frac{D^{3} f_{\alpha}(x)[h, h, h]}{f_{\alpha}(x)}\right\}\right. \\
& \left.+(p-1)\left\{(p-2) \mathbf{E}_{x}\left\{\sigma_{x, h}^{3}(\cdot)\right\}-3 \mathbf{E}_{x}\left\{S_{x, h}(\cdot)\right\} \mathbf{E}_{x}\left\{\sigma_{x, h}^{2}(\cdot)\right\}+3 \mathbf{E}_{x}\left\{\sigma_{x, h}(\cdot) \cdot \frac{D^{2} f_{\alpha}(x)[h, h]}{f_{\alpha}(x)}\right\}\right\}\right] .
\end{aligned}
$$

Proposition 5.1 Suppose $p \geq 1$ and that $D^{2} f_{\alpha}(x)[h, h] \geq 0$ for every $x \in \mathbb{R}_{++}^{n}$ and for every $h \in \mathbb{R}^{n}$. Let $\xi_{1}$ and $\xi_{2}$ be given such that

$$
\sup _{x \in \mathbb{R}_{++}^{n} ; h:(x \pm h) \geq 0}\left\{\left|S_{x, h}\right|\right\} \leq \xi_{1},
$$

and

$$
\left|D^{3} f_{\alpha}(x)[h, h, h]\right| \leq \xi_{2} D^{2} f_{\alpha}(x)[h, h], \quad \text { for every } x \in \mathbb{R}_{++}^{n}, \text { and } h \text { such that }(x \pm h) \geq 0 .
$$

Then, for every $x \in \mathbb{R}_{++}^{n}$ and every $h \in \mathbb{R}^{n}$ such that $(x \pm h) \geq 0$, we have

$$
\left|D^{3} \Phi(x)[h, h, h]\right| \leq \max \left\{(2|p-2|+3) \xi_{1}, 6(p-1) \xi_{1}+\xi_{2}\right\} D^{2} \Phi(x)[h, h] .
$$

Proof. We simply substitute the bounds given in the assumption part of the statement in the expression for the third derivative (given immediately before the proposition) and the claim of the proposition easily follows. 


\section{Recovering a Good Dual Solution}

In the previous sections, we showed how to construct self-concordant barriers for the convex approximations $\tilde{G}$ of the convex set of main interest $G$. Once we have such a barrier, we can use the general self-concordance theory and we immediately have various path-following and potential-reduction interior-point algorithms to optimize a linear function over $\tilde{G}$.

If we can compute the Legendre-Fenchel conjugate of our barrier efficiently, then we can even apply some primal-dual algorithms (as in [15]). However, if the Legendre-Fenchel conjugate is not available for efficient computation, then we are stuck with primal-only algorithms. Even in such a case, we would be interested in generating good dual solutions. This section is dedicated to showing a way to recover a good dual solution from a good, central primal solution. Given a central primal solution of a convex optimization problem in conic form together with a logarithmically-homogeneous s.c.b. we can generate a central dual solution using a negative multiple of the gradient of the s.c.b. (this is well-known). Here, we work out in some detail the duals of the convex optimization problems arising from our $p$-norm constructions to facilitate the further development of primal-dual approaches based on the approximation schemes that we proposed.

We start with the polyhedral case. Let $A$ be as in Section 2 and for $p \geq 1$, consider

$$
\mathbf{K}(p):=\left\{(t, x) \in \mathbb{R} \times \mathbb{R}_{+}^{n}:\|A x\|_{p} \leq t\right\} .
$$

We define $q$ by $\frac{1}{p}+\frac{1}{q}=1$. Then

$$
[\mathbf{K}(p)]^{*}=\left\{(\tau, \xi) \in \mathbb{R} \times \mathbb{R}_{+}^{n}: A^{T} \eta+\xi \geq 0,\|\eta\|_{q} \leq \tau, \text { for some } \eta \in \mathbb{R}^{m}\right\} .
$$

We use the s.c.b.

$$
F_{p}(t, x):=-\ln (t-H(x))-\left(\frac{2|p-2|+3}{3}\right)^{2} \sum_{j=1}^{n} \ln \left(x_{j}\right) .
$$

Now, let us consider the primal optimization problem

$$
\max \left\{e^{T} x:\|A x\|_{p} \leq 1, x \geq 0\right\} .
$$

The dual is

$$
\min \left\{\tau: A^{T} \eta \geq e,\|\eta\|_{q} \leq \tau, \text { for some } \eta \in \mathbb{R}^{m}\right\} .
$$

For $\rho>0$, let $x_{\rho}:=\operatorname{argmin}\left\{\rho\left(-e^{T} x\right)+F_{p}(1, x)\right\}$ (that is, $x_{\rho}$ is the central primal solution corresponding to $\rho$ ). Then we conclude

$$
-\rho e-\left(\frac{2|p-2|+3}{3}\right)^{2}\left[\operatorname{diag}\left(x_{\rho}\right)\right]^{-1}+\frac{1}{\left\|A x_{\rho}\right\|_{p}^{p-1}\left(1-\left\|A x_{\rho}\right\|_{p}\right)} A^{T}\left[A x_{\rho}\right]^{p-1}=0 .
$$

Setting $\eta_{\rho}:=\frac{1}{\rho\left\|A x_{\rho}\right\|_{p}^{p-1}\left(1-\left\|A x_{\rho}\right\|_{p}\right)}\left[A x_{\rho}\right]^{p-1}$ yields $\left\|\eta_{\rho}\right\|_{q}=\frac{1}{\rho\left(1-\left\|A x_{\rho}\right\|_{p}\right)}$. Thus, $\eta_{\rho}$ is feasible in the dual with $\tau_{\rho}:=\frac{1}{\rho\left(1-\left\|A x_{\rho}\right\|_{p}\right)}$. Indeed, by taking $\tau$ slightly larger, we can also easily generate an interior point for the dual problem (we already have $A^{T} \eta_{\rho}>e$ ) without sacrificing much in the dual objective value.

We can exploit the nice duality between $p$-norm cones and $q$-norm cones in more generality. To move towards the set-up of Section 4, let us give a simple example to see the derivation of the dual problem. 
Example. Let $Q_{i} \succeq 0$. Then consider

$$
\begin{aligned}
& \min _{x}\left\{-e^{T} x:\left\|\lambda\left(\sum_{i} x_{i} Q_{i}\right)\right\|_{p} \leq 1, x \geq 0\right\} \\
& \Downarrow \\
& \max _{\phi, \tau, \eta}\left\{\begin{array}{ll} 
& \phi_{i}-\operatorname{Tr}\left(\eta Q_{i}\right)=-1, \forall i \\
-\tau: & \phi \geq 0 \\
\|\lambda(\eta)\|_{q} \leq \tau
\end{array}\right\} \\
& \mathbb{1} \\
& -\min _{\eta}\left\{\|\lambda(\eta)\|_{q}: \operatorname{Tr}\left(\eta Q_{i}\right) \geq 1, \forall i\right\} \\
& \mathbb{\Downarrow} \\
& -\min _{\eta}\left\{\|\lambda(\eta)\|_{q}: \operatorname{Tr}\left(\eta Q_{i}\right) \geq 1, \forall i, \eta \succeq 0\right\} .
\end{aligned}
$$

Now, we are ready to move to the full generality of the set-up of Section 4 .

Cones $\mathbf{M}_{p}$. First, we assume that the primal problem involves the cones $\mathbf{M}_{p}$ and derive the dual problem. Then, we work out exact expressions for a good dual solution corresponding to a given good primal solution. For $y \in \mathbb{S}^{m}$ and $p \in[1, \infty]$, let $|y|_{p}:=\|\lambda(y)\|_{p}$. Further let $\mathbf{K}$ be a closed convex cone with nonempty interior in an Euclidean space $(\mathbb{E},\langle\cdot, \cdot\rangle)$ and $\mathcal{P}$ be a linear mapping from $\mathbb{E}$ to $\mathbb{S}^{m}$ such that $\mathcal{P} x \succ 0$ whenever $x \in \operatorname{int} \mathbf{K}$. Finally, we define

$$
\mathbf{M}_{p}:=\left\{(t, x): x \in \mathbf{K},|\mathcal{P} x|_{p} \leq t\right\} .
$$

Now, we work out the cone dual to $\mathbf{M}_{p}$. Note that the primal cone $\mathbf{M}_{p}$ can be written as the intersection of two cones: $(\mathbb{R} \times \mathbf{K})$ and a linear image of the $p$-norm cone. By our assumption above, the relative interiors of these two cones intersect. Therefore, the dual of the intersection is the Minkowski sum of the duals of the two cones. We compute $\left(\langle\cdot, \cdot\rangle_{F}\right.$ stands for the Frobenius inner product on $\left.\mathbb{S}^{m}\right)$ :

$$
\begin{aligned}
\mathbf{M}_{p}^{*} & =\left\{(\tau, \xi): x \in \mathbf{K},|\mathcal{P} x|_{p} \leq t \Rightarrow \tau t+\langle x, \xi\rangle \geq 0\right\} \\
& =\left\{(\tau, \xi): \exists\left(\eta \in \mathbb{S}^{n}, \phi \in \mathbf{K}^{*}\right) \text { such that } \xi=\phi-\mathcal{P}^{*} \eta,|\eta|_{q} \leq \tau\right\} .
\end{aligned}
$$

Since $\mathcal{P}$ maps $\mathbf{K}$ into $\mathbb{S}_{+}^{m}, \mathcal{P}^{*}$ maps $\mathbb{S}_{+}^{m}$ into $\mathbf{K}^{*}$; thus, whenever $\eta^{\prime} \succeq \eta$, we have $\left(\mathcal{P}^{*} \eta^{\prime}-\mathcal{P}^{*} \eta\right) \in$ $\mathbf{K}^{*}$. It follows that if $\xi=\phi-\mathcal{P}^{*} \eta$ with $\phi \in \mathbf{K}^{*}$ and $|\eta|_{p} \leq \tau$ and $\eta_{+}$is the "positive part" of $\eta$, then $\xi=\underbrace{\left(\phi+\mathcal{P}^{*} \eta_{+}-\mathcal{P}^{*} \eta\right)}_{\in \mathbf{K}^{*}}-\mathcal{P}^{*} \eta_{+}$and $\left|\eta_{+}\right|_{q} \leq \tau$. We arrive at

$$
\mathbf{M}_{p}^{*}=\left\{(\tau, \xi): \exists\left(\phi \in \mathbf{K}^{*}, \eta \succeq 0\right) \text { such that } \xi=\phi-\mathcal{P}^{*} \eta,|\eta|_{q} \leq \tau\right\} .
$$

Thus, a primal-dual pair of conic problems associated with $\mathbf{M}_{p}$ is

$$
\begin{aligned}
& \min _{s}\left\{e^{T} s: A s-b \in \mathbf{K},|\mathcal{P}(A s-b)|_{p} \leq c^{T} s-d\right\} \\
& \mathbb{1} \\
& \max _{\phi, \eta, \tau}\left\{\left\langle\phi-\mathcal{P}^{*} \eta, b\right\rangle+\tau d: A^{*}\left(\phi-\mathcal{P}^{*} \eta\right)+\tau c=e, \phi \in \mathbf{K}^{*},|\eta|_{q} \leq \tau, \eta \succeq 0\right\} .
\end{aligned}
$$

Here, the data are given by $(A, b, c, d, e)$, where $e$ (no longer a vector of all ones) is arbitrary. 
Now, let $p \geq 2$ be integer, $F$ be a $\vartheta$-logarithmically homogeneous s.c.b. for $\mathbf{K}, \alpha \geq 1$ and $\beta=O(1) p ;$ then

$$
\Phi_{p}(t, x)=-\alpha \ln \left(t-|\mathcal{P} x|_{p}\right)+\beta^{2} F(x)
$$

is $\vartheta_{p}$-logarithmically homogeneous s.c.b. for $\mathbf{M}_{p}$, with

$$
\vartheta_{p}:=\alpha+\beta^{2} \vartheta
$$

Let $s_{\rho}$ be a central solution to $(P): s_{\rho}:=\underset{s}{\operatorname{argmin}}\left\{\rho e^{T} s+\Phi_{p}\left(c^{T} s-d, A s-b\right)\right\}$. Then we define

$$
x_{\rho}:=A s_{\rho}-b, t_{\rho}:=c^{T} s_{\rho}-d, \zeta_{\rho}:=\mathcal{P} x_{\rho}, \omega_{\rho}:=\left|\zeta_{\rho}\right|_{p}, \xi_{\rho}:=\left[\omega_{\rho}^{-1} \zeta_{\rho}\right]^{p-1} .
$$

Thus, we have

$$
\rho e-\frac{\alpha}{t_{\rho}-\omega_{\rho}}\left[c-A^{*} \mathcal{P}^{*} \xi_{\rho}\right]+\beta^{2} A^{*} \nabla F\left(A s_{\rho}-b\right)=0 .
$$

Upon further defining,

$$
\tau_{\rho}:=\frac{\alpha}{\rho\left(t_{\rho}-\omega_{\rho}\right)}, \eta_{\rho}:=\tau_{\rho} \xi_{\rho}, \phi_{\rho}:=-\frac{\beta^{2}}{\rho} \nabla F\left(A s_{\rho}-b\right), \lambda:=\lambda\left(\xi_{\rho}\right)\left[\left|\omega_{\rho}^{-1} \zeta_{\rho}\right|_{p}=1 \Rightarrow\|\lambda\|_{\frac{p}{p-1}}=1\right],
$$

we conclude

$$
\left\{\begin{array}{c}
A^{*}\left(\phi_{\rho}-\mathcal{P}^{*} \eta_{\rho}\right)+\tau_{\rho} c=e, \phi_{\rho} \in \mathbf{K}^{*}, \eta_{\rho} \succeq 0 \\
\left|\eta_{\rho}\right|_{q}=\tau_{\rho}\|\lambda\|_{\frac{p}{p-1}}=\tau_{\rho}
\end{array}\right\}
$$

That is, a central solution $s_{\rho}$ generates a feasible solution $\left(\phi_{\rho}, \eta_{\rho}, \tau_{\rho}\right)$ of $(D)$. We have

$$
\begin{aligned}
& \left\langle\phi_{\rho}-\mathcal{P}^{*} \eta_{\rho}, b\right\rangle+\tau_{\rho} d=\overbrace{\left\langle\phi_{\rho}-\mathcal{P}^{*} \eta_{\rho}, A s_{\rho}\right\rangle+\tau_{\rho} c^{T} s_{\rho}}^{=s_{\rho}^{T}\left[A^{*}\left(\phi_{\rho}-\mathcal{P}^{*} \eta_{\rho}\right)+\tau_{\rho} c\right]=s_{\rho}^{T} e}+\left\langle\phi_{\rho}-\mathcal{P}^{*} \eta_{\rho}, b-A s_{\rho}\right\rangle+\tau_{\rho}\left(d-c^{T} s_{\rho}\right) \\
& =e^{T} s_{\rho}+\frac{1}{\rho}\left[\nabla \Phi_{p}\left(c^{T} s_{\rho}-d, A s_{\rho}-b\right)\right]^{T}\left[\begin{array}{c}
c^{T} s_{\rho}-d \\
A s_{\rho}-b
\end{array}\right]=e^{T} s_{\rho}-\frac{\vartheta(p)}{\rho} .
\end{aligned}
$$

This completes the derivation of the dual problems for $\mathbf{M}_{p}$ cones and the computation of good dual solutions. Next, we perform the analogous derivation for $\mathbf{N}_{p}$ cones.

Cones $\mathbf{N}_{p}$. Let $\mathbf{K}$ be a closed convex cone with a nonempty interior in Euclidean space $(\mathbb{E},\langle\cdot, \cdot\rangle)$ and $\mathcal{P}$ be a linear mapping from $\mathbb{E}$ to $\mathbb{S}^{m}$ such that $\mathcal{P} x \succ 0$ whenever $x \in \operatorname{int} \mathbf{K}$. For positive integer $p \geq 2$, let

$$
\mathbf{N}_{p}:=\operatorname{cl}\left\{(t, x): t>0, x \in \operatorname{int} \mathbf{K},\left(\operatorname{Tr}\left([\mathcal{P} x]^{-p}\right)\right)^{-1 / p} \geq t\right\} .
$$


We have

$$
\begin{aligned}
& \mathbf{N}_{p}^{*}=\left\{(\tau, \xi): x \in \operatorname{int} \mathbf{K}, t>0,\left|[\mathcal{P} x]^{-1}\right|_{p} \leq t^{-1} \Rightarrow \tau t+\langle\xi, x\rangle \geq 0\right\} \\
& =\left\{(\tau, \xi): x \in \operatorname{int} \mathbf{K}, t>0,\left|t^{2}[\mathcal{P} x]^{-1}\right|_{p} \leq t \Rightarrow \tau t+\langle\xi, x\rangle \geq 0\right\} \\
& =\left\{(\tau, \xi): \min _{x, y, t}\left\{\tau t+\langle\xi, x\rangle: t^{2}[\mathcal{P} x]^{-1} \preceq y,|y|_{p} \leq t, x \in \operatorname{int} \mathbf{K}, t>0\right\} \geq 0\right\} \\
& =\left\{(\tau, \xi): \min _{x, y, t}\left\{\tau t+\langle\xi, x\rangle:\left[\begin{array}{cc}
y & t I \\
t I & \mathcal{P} x
\end{array}\right] \succeq 0,|y|_{p} \leq t, t>0, x \in \operatorname{int} \mathbf{K}\right\} \geq 0\right\} \\
& =\left\{(\tau, \xi): \min _{x, y, t}\left\{\tau t+\langle\xi, x\rangle:\left[\begin{array}{cc}
y & t I \\
t I & \mathcal{P} x
\end{array}\right] \succeq 0,|y|_{p} \leq t, x \in \mathbf{K}\right\} \geq 0\right\} \\
& =\left\{(\tau, \xi): \exists(\alpha, \beta, \eta, \gamma, \sigma, \phi) \text { s.t. } \begin{array}{c}
{\left[\begin{array}{cc}
\alpha & \beta^{T} \\
\beta & \eta
\end{array}\right] \succeq 0,|\gamma|_{q} \leq \sigma, \phi \in \mathbf{K}^{*},} \\
\operatorname{Tr}(y \alpha)+2 \operatorname{Tr}(t \beta)+\operatorname{Tr}(\eta \mathcal{P} x)+t \sigma-\operatorname{Tr}(y \gamma)+\langle\phi, x\rangle \\
=\tau t+\langle\xi, x\rangle \forall x, y, t
\end{array}\right\} \\
& =\left\{(\tau, \xi): \exists\left(\eta \succeq 0, \phi \in \mathbf{K}^{*}, \sigma\right) \text { s.t. } \quad \begin{array}{ll}
\xi=\phi+\mathcal{P}^{*} \eta \\
& |\sigma-\tau| \leq 2 \max _{\alpha, \beta}\left\{2 \operatorname{Tr}(\beta):\left[\begin{array}{cc}
\alpha & \beta^{T} \\
\beta & \eta
\end{array}\right] \succeq 0,|\alpha|_{q} \leq \sigma\right\}
\end{array}\right\} .
\end{aligned}
$$

To simplify the last expression, let us solve the optimization problem

$$
\max _{\alpha, \beta}\left\{2 \operatorname{Tr}(\beta):\left[\begin{array}{cc}
\alpha & \beta^{T} \\
\beta & \eta
\end{array}\right] \succeq 0,|\alpha|_{q} \leq \sigma\right\} .
$$

When solving the problem, we may assume without loss of generality that $\eta$ is diagonal. In this case, the feasible solution set of the problem remains invariant under the mappings $(\alpha, \beta) \mapsto$ $(G \alpha G, G \beta G)$, where $G$ is a diagonal matrix with diagonal entries \pm 1 , and the objective function also remains invariant under these mappings. Since the problem is convex, it follows that the optimal value remains unchanged when $\alpha, \beta$ are restricted to be invariant with respect to the above transformations (that is, we can assume that $\alpha$ and $\beta$ are diagonal). In this case, the problem becomes

$$
\mathrm{Opt}=\max _{\left\{\alpha_{i}\right\},\left\{\beta_{i}\right\}}\left\{\sum_{i} \beta_{i}: \beta_{i}^{2} \leq \alpha_{i} \eta_{i}, \sum_{i} \alpha_{i}^{\frac{p}{p-1}} \leq \sigma^{\frac{p}{p-1}}\right\},
$$

where $\eta_{i}$ are the eigenvalues of $\eta$. We have

$$
\begin{aligned}
& \mathrm{Opt}=\max _{\alpha_{i}}\left\{\sum_{i} \eta_{i}^{1 / 2} \alpha_{i}^{1 / 2}: \sum_{i} \alpha_{i}^{\frac{p}{p-1}} \leq \sigma^{\frac{p}{p-1}}\right\}=\max _{\delta_{i}}\left\{\sum_{i} \delta_{i} \eta_{i}^{1 / 2}:\|\delta\|_{\frac{2 p}{p-1}} \leq \sigma^{1 / 2}\right\}
\end{aligned}
$$

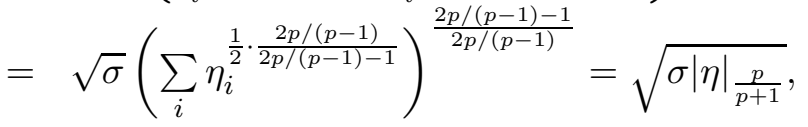

where $\eta \in \mathbb{S}_{+}^{m}$; note that $|\eta|_{r}$ is concave in $\eta \in \mathbb{S}_{+}^{m}$ when $0<r \leq 1$. Thus,

$$
\mathbf{N}_{p}^{*}=\left\{(\tau, \xi): \exists\left(\eta \succeq 0, \phi \in \mathbf{K}^{*}, \sigma \geq 0\right) \text { such that } \xi=\phi+\mathcal{P}^{*} \eta,|\tau-\sigma| \leq 2 \sqrt{\sigma|\eta|_{\frac{p}{p+1}}}\right\} .
$$

Let us focus on the set $\left\{\tau: \exists \sigma \geq 0,|\tau-\sigma| \leq 2 \sqrt{\sigma|\eta|_{\frac{p}{p+1}}}\right\}$. The only constraint on $\tau$ implied by the above is

$$
\exists \sigma \geq 0: \quad \tau \geq \sigma-2 \sqrt{\sigma|\eta|_{\frac{p}{p+1}}}=\left(\sqrt{\sigma}-\sqrt{|\eta|_{\frac{p}{p+1}}}\right)^{2}-|\eta|_{\frac{p}{p+1}} .
$$


Equivalently, $\tau \geq-|\eta|_{\frac{p}{p+1}}$. We deduce,

$$
\mathbf{N}_{p}^{*}=\left\{(\tau, \xi): \exists\left(\eta \succeq 0, \phi \in \mathbf{K}^{*}\right) \text { such that } \xi=\phi+\mathcal{P}^{*} \eta, 0 \leq \tau+|\eta|_{\frac{p}{p+1}}\right\} \text {. }
$$

Thus, a primal-dual pair of conic problems associated with $\mathbf{N}_{p}$ is

$$
\begin{aligned}
& \min _{s}\left\{e^{T} s: A s-b \in \mathbf{K}, c^{T} s-d \geq 0,\left|[\mathcal{P}(A s-b)]^{-1}\right|_{p} \geq \frac{1}{c^{T} s-d}\right\} \\
& \mathbb{\Downarrow} \\
& \max _{\phi, \eta, \tau}\left\{\left\langle\phi+\mathcal{P}^{*} \eta, b\right\rangle+\tau d: A^{*}\left(\phi+\mathcal{P}^{*} \eta\right)+\tau c=e, \phi \in \mathbf{K}^{*}, 0 \leq \tau+|\eta|_{\frac{p}{p+1}}, \eta \succeq 0\right\} .
\end{aligned}
$$

Now, let $F$ be a $\vartheta$-logarithmically homogeneous s.c.b. for $\mathbf{K}, \alpha, \gamma \geq 1$ and $\beta=O(1) p^{2}$; then

$$
\Psi_{p}(t, x)=-\alpha \ln \left(\left|[\mathcal{P} x]^{-1}\right|_{p}^{-1}-t\right)-\gamma \ln t+\beta^{2} F(x)
$$

is $\vartheta_{p}$-logarithmically homogeneous s.c.b. for $\mathbf{N}_{p}$, with

$$
\vartheta_{p}:=\alpha+\gamma+\beta^{2} \vartheta
$$

Let $s_{\rho}$ be a central solution to $(P): s_{\rho}:=\operatorname{argmin}\left\{\rho e^{T} s+\Psi_{p}\left(c^{T} s-d, A s-b\right)\right\}$. Then, we define

$$
x_{\rho}:=A s_{\rho}-b, t_{\rho}:=c^{T} s_{\rho}-d, \zeta_{\rho}:=\left[\mathcal{P} x_{\rho}\right]^{-1}, \omega_{\rho}:=\left|\zeta_{\rho}\right|_{p}, \xi_{\rho}:=\left[\omega_{\rho}^{-1} \zeta_{\rho}\right]^{p+1} .
$$

Thus, with these definitions, we have

$$
\rho e-\frac{\alpha}{\omega_{\rho}^{-1}-t_{\rho}}\left[A^{*} \mathcal{P}^{*} \xi_{\rho}-c\right]-\frac{\gamma}{t_{\rho}} c+\beta^{2} A^{*} \nabla F\left(A s_{\rho}-b\right)=0 .
$$

Further defining

$$
\begin{gathered}
\delta_{\rho}:=\frac{\alpha}{\rho\left(\omega_{\rho}^{-1}-t_{\rho}\right)}, \tau_{\rho}:=\frac{\gamma}{\rho t_{\rho}}-\delta_{\rho}, \eta_{\rho}:=\delta_{\rho} \xi_{\rho}, \\
\phi_{\rho}:=-\frac{\beta^{2}}{\rho} \nabla F\left(A s_{\rho}-b\right), \lambda:=\lambda\left(\xi_{\rho}\right)\left[\left|\omega_{\rho}^{-1} \zeta_{\rho}\right|_{p}=1 \Rightarrow\|\lambda\|_{\frac{p}{p+1}}=1\right],
\end{gathered}
$$

we deduce

$$
\left\{\begin{array}{c}
A^{*}\left(\phi_{\rho}+\mathcal{P}^{*} \eta_{\rho}\right)+\tau_{\rho} c=e, \phi \in \mathbf{K}^{*}, \eta \succeq 0 \\
\left|\eta_{\rho}\right|_{\frac{p}{p+1}}=\delta_{\rho}\|\lambda\|_{\frac{p}{p+1}}=\delta_{\rho} \geq-\tau_{\rho}
\end{array}\right\} .
$$

So, a central solution $s_{\rho}$ generates a feasible solution $\left(\phi_{\rho}, \eta_{\rho}, \tau_{\rho}\right)$ of $(D)$. We have

$$
\begin{aligned}
\left\langle\phi_{\rho}+\mathcal{P}^{*} \eta_{\rho}, b\right\rangle+\tau_{\rho} d & =\overbrace{\left\langle\phi_{\rho}+\mathcal{P}^{*} \eta_{\rho}, A s_{\rho}\right\rangle+\tau_{\rho} c^{T} s_{\rho}}^{=s_{\rho}^{T}\left[A^{*}\left(\phi_{\rho}+\mathcal{P}^{*} \eta_{\rho}\right)+\tau_{\rho} c\right]=s_{\rho}^{T} e}+\left\langle\phi_{\rho}+\mathcal{P}^{*} \eta_{\rho}, b-A s_{\rho}\right\rangle+\tau_{\rho}\left(d-c^{T} s_{\rho}\right) \\
& =e^{T} s_{\rho}+\frac{1}{\rho}\left[\nabla \Psi_{p}\left(c^{T} s_{\rho}-d, A s_{\rho}-b\right)\right]^{T}\left[\begin{array}{c}
c^{T} s_{\rho}-d \\
A s_{\rho}-b
\end{array}\right]=e^{T} s_{\rho}-\frac{\vartheta(p)}{\rho} .
\end{aligned}
$$




\section{Lipschitz Continuous Gradients}

For the minimization of convex functions with Lipschitz continuous gradients, under certain favorable circumstances, the first-order methods can achieve the further improved iteration bound of $O\left(\sqrt{\frac{\gamma}{\epsilon}}\right)$ (see [20], also see [13]), where $\gamma$ is the Lipschitz constant for the gradient of the convex function. Instead of solving

$$
\max _{x}\left\{\langle c, x\rangle:\left(\sum_{i}\left\langle a_{i}, x\right\rangle^{p}\right)^{1 / p} \leq 1\right\}
$$

we may consider solving the unconstrained problem

$$
\min _{x}\left\{-\langle c, x\rangle+\xi\left[\left(\sum_{i}\left\langle a_{i}, x\right\rangle^{p}\right)^{1 / p}-1\right]\right\},
$$

where $\xi(\cdot)$ is a penalty function such that the above function (as a function of $x$ ) is convex and has Lipschitz continuous gradient with a modest Lipschitz constant. Keeping this connection in mind, recall from Section 2 that the barrier parameters of the new barriers were bounded from above by $\Theta\left(\frac{1}{\epsilon^{2}}\right)$ functions yielding only $\Theta\left(\frac{1}{\epsilon}\right)$ upper bounds on the iteration complexity. In this context, we may ask "is there any hope of improving the $\Theta\left(\frac{1}{\epsilon}\right)$ iteration complexity bound for our approach to perhaps $\Theta\left(\sqrt{\frac{\gamma}{\epsilon}}\right)$ under some conditions analogous to the conditions for the first-order algorithms for unconstrained convex minimization?" So, continuing the theme from Sections 4 and 6 , let us look at a $p$-norm type function applied to the eigenvalues of a symmetric matrix. The next result suggests that the $p$-norm construction is well-behaved in this regard and that there is some hope for further positive results.

Let $p \geq 3$, and let

$$
H(x):=\left\|\lambda_{+}(x)\right\|_{p}^{2}, S(x):=\left\|\lambda_{+}(x)\right\|_{p}^{p},
$$

where $x$ is a symmetric matrix and $\lambda_{+}(x)$ is the vector with the entries $\max \left\{0, \lambda_{i}(x)\right\}$ and $\lambda_{i}(x)$ are the eigenvalues of $x$.

Proposition 7.1 The function $H(\cdot)$ is convex and continuously differentiable with Lipschitz continuous gradient, specifically,

$$
x, y \in \mathbb{S}^{n} \Rightarrow\left|H^{\prime}(x)-H^{\prime}(y)\right|_{q} \leq 2(p-1)|x-y|_{p} .
$$

Proof. It suffices to verify that $H$ is continuously differentiable, twice continuously differentiable (except at the origin) such that

$$
x \neq 0 \Rightarrow 0 \leq D^{2} H(x)[h, h] \leq 2(p-1)|h|_{p}^{2} \forall h .
$$

Let $\gamma$ be a simple closed curve in the right half-plane which encircles $[0, L]$. For $x \in \mathbb{S}^{n}$ with $\max _{i} \lambda_{i}(x)<L$ we have

$$
S(x)=\frac{1}{2 \pi i} \oint_{\gamma} z^{p} \operatorname{Tr}\left((z I-x)^{-1}\right) d z
$$

whence, as it is immediately seen, $S$ is twice continuously differentiable everywhere, so that $H$ is twice continuously differentiable (except at the origin); moreover, $H$ clearly is continuously 
differentiable. It is also well-known that $H$ is convex (as a symmetric convex function of the eigenvalues of a symmetric matrix).

Let $\lambda_{i}$ be the eigenvalues of $x, J_{+}=\left\{i: \lambda_{i}>0\right\}, J_{-}=\left\{i: \lambda_{i} \leq 0\right\}$, let $h$ be a symmetric matrix and $\bar{h}_{i j}$ be the entries of $h$ in the orthonormal eigenbasis of $x$. Then, by differentiating under the integral and using integration by parts, we arrive at

$$
\begin{aligned}
D S(x)[h] & =\frac{1}{2 \pi i} \oint_{\gamma} p z^{p-1} \operatorname{Tr}\left((z I-x)^{-1} h\right) d z=p \sum_{i \in J_{+}} \lambda_{i}^{p-1} \bar{h}_{i i} \\
D^{2} S(x)[h, h] & =\frac{1}{2 \pi i} \oint_{\gamma} p z^{p-1} \operatorname{Tr}\left((z I-x)^{-1} h(z I-x)^{-1} h\right) d z=\frac{1}{2 \pi i} \oint_{\gamma} p z^{p-1}\left(\sum_{i, j} \bar{h}_{i j}^{2} \frac{1}{\left(z-\lambda_{i}\right)\left(z-\lambda_{j}\right)}\right) d z \\
& =p \sum_{i, j \in J_{+}} \bar{h}_{i j}^{2} \frac{\lambda_{i}^{p-1}-\lambda_{j}^{p-1}}{\lambda_{i}-\lambda_{j}}+2 p \sum_{i \in J_{+}, j \in J_{-}} \bar{h}_{i j}^{2} \frac{\lambda_{i}^{p-1}}{\lambda_{i}-\lambda_{j}}\left[\text { where } \frac{a^{p-1}-b^{p-1}}{a-b}=(p-1) a^{p-2} \text { when } a=b\right] \\
& \leq p(p-1) \sum_{i, j \in J_{+}} \bar{h}_{i j}^{2} \frac{\lambda_{i}^{p-2}+\lambda_{j}^{p-2}}{2}+2 p \sum_{i \in J_{+}, j \in J_{-}} \bar{h}_{i j}^{2} \lambda_{i}^{p-2}\left[\text { due to } \frac{\theta^{q}-1}{\theta-1} \leq q \frac{\theta^{q-1}+1}{2} \text { for } \theta, q \geq 1\right] \\
& \leq p(p-1) \sum_{i \in J_{+}} \lambda_{i}^{p-2} \sum_{j} \bar{h}_{i j}^{2}
\end{aligned}
$$

whence

$$
\begin{aligned}
D H(x)[h] & =\frac{2}{p}(S(x))^{\frac{2}{p}-1} D S(x)[h]=2(S(x))^{\frac{2}{p}-1} \sum_{i \in J_{+}} \bar{h}_{i i} \lambda_{i}^{p-1} \\
D^{2} H[x][h, h] & =\frac{2}{p}\left(\frac{2}{p}-1\right)(S(x))^{\frac{2}{p}-2}(D S(x)[h])^{2}+\frac{2}{p}(S(x))^{\frac{2}{p}-1} D^{2} S(x)[h, h] \\
& \leq \frac{2}{p}(S(x))^{\frac{2}{p}-1} D^{2} S(x)[h, h] .
\end{aligned}
$$

Since $D^{2} H(x)[h, h]$ is homogeneous of degree 0 with respect to $x$, we may assume when computing $D^{2} H(x)[h, h]$ that $S(x)=1$, that is, $\sum_{i \in J_{+}} \lambda_{i}^{p}=1$. In this case, setting $\eta_{i}=\sqrt{\sum_{j} \bar{h}_{i j}^{2}}$ :

$$
\begin{aligned}
D^{2} H(x)[h, h] & \leq \frac{2}{p} D^{2} S(x)[h, h] \leq 2(p-1) \sum_{i \in J_{+}} \lambda_{i}^{p-2} \eta_{i}^{2} \leq 2(p-1)\left(\sum_{i \in J_{+}} \lambda_{i}^{p}\right)^{\frac{p-2}{p}}\left(\sum_{i \in J_{+}} \eta_{i}^{p}\right)^{\frac{2}{p}} \\
& \leq 2(p-1)|h|_{p}^{2},
\end{aligned}
$$

where the concluding inequality is due to the following observation:

For $h \in \mathbb{S}^{n}$ and $p \geq 2$, let $\eta$ be the vector with entries equal to the Euclidean lengths of the columns in $h$. Then $\|\eta\|_{p} \leq|h|_{p}$.

Indeed, setting $h=v s v^{T}$, where $v$ is orthogonal and $s$ is diagonal with diagonal entries $s_{i}$, the Euclidean norms of the columns in $h$ are the same as Euclidean norms of the columns in $s v^{T}: \eta_{j}^{2}=\sum_{i} s_{i}^{2} v_{j i}^{2}$. In other words, the vector $[\eta]^{2}:=$ $\left(\eta_{1}^{2}, \eta_{2}^{2}, \ldots, \eta_{n}^{2}\right)$ is obtained from the vector $[s]^{2}=\left(s_{1}^{2}, s_{2}^{2}, \ldots, s_{n}^{2}\right)$ by multiplication by a doubly-stochastic matrix. It follows that $\|\eta\|_{p}^{2}=\left\|[\eta]^{2}\right\|_{p / 2} \leq\left\|[s]^{2}\right\|_{p / 2}=\|s\|_{p}^{2}$, as claimed. Note that we used the classical inequality $\left\|[\eta]^{2}\right\|_{p / 2} \leq\left\|[s]^{2}\right\|_{p / 2}$ which goes back to Hardy, Littlewood and Pólya.

We have demonstrated (24).

\section{Conclusion and Future Work}

There are four clear research directions motivated by this work: 
1. Design and analysis of cutting-plane interior-point algorithms based on the self-concordant barriers constructed here. One major advantage of our barriers over those used in the preexisting work $([1,2,8,12])$ is that we do not need to drop any constraints and the addition of new constraints does not change the barrier parameter $\vartheta$.

2. Further extension of the theory to constraints defined by other partial orders, cones (e.g., partial orders induced by hyperbolic cones [23]).

3. Improvement of the iteration complexity bound from $O\left(\frac{1}{\epsilon}\right)$ to $O\left(\sqrt{\frac{\gamma}{\epsilon}}\right)$ under some favorable conditions, as suggested in Section 7.

4. Improvement of the computational complexity of evaluating $f, f^{\prime}$ and $f^{\prime \prime}$ for such selfconcordant barriers.

\section{References}

[1] K. M. Anstreicher, Towards a practical volumetric cutting plane method for convex programming, SIAM J. Optim. 9, 190-206 (1999).

[2] D. S. Atkinson, P. M. Vaidya, A cutting plane algorithm for convex programming that uses analytic centers, Math. Prog. 69, 1-43 (1995).

[3] A. Ben-Tal, A. Nemirovski, Lectures on Modern Convex Optimization (MPS-SIAM Series on Optimization, SIAM, Philadelphia, 2001).

[4] D. Bienstock, Potential Function Methods for Approximately Solving Linear Programming Problems. Theory and Practice (Kluwer Academic Publishers, Boston, USA, 2002).

[5] D. Bienstock, G. Iyengar, Approximating fractional packings and coverings in $O(1 / \epsilon)$ iterations, SIAM J. Comput. 35, 825-854 (2006).

[6] G. Cornuéjols, Combinatorial Optimization, Packing and Covering (CBMS-NSF Regional conference series in applied mathematics, SIAM, 2001).

[7] N. Garg, J. Könemann, Faster and simpler algorithms for multicommodity flow and other fractional packing problems, Proc. 39th Ann. Symp. on FOCS 300-309 (1998).

[8] J.-L. Goffin, Z.-Q. Luo, Y. Ye, Complexity analysis of an interior cutting plane method for convex feasibility problems, SIAM J. Optim. 6, 638-652 (1996).

[9] M. D. Grigoriadis, L. G. Khachiyan, Coordination complexity of parallel price-directive decomposition, Math. Oper. Res. 21, 321-340 (1996).

[10] P. Klein, S. A. Plotkin, C. Stein, É. Tardos, Faster approximation algorithms for the unit capacity concurrent flow problem with applications to routing and finding sparse cuts, SIAM J. Computing 23, 466-487 (1994).

[11] T. Leighton, F. Makedon, S. A. Plotkin, C. Stein, É. Tardos, S. Tragoudas, Fast approximation algorithms for multicommodity flow problems, J. Comput. System Sciences 50, 228-243 (1995). 
[12] J. E. Mitchell, M. J. Todd, Solving combinatorial optimization problems using Karmarkar's algorithm, Math. Prog. 56, 245-284 (1992).

[13] A. Nemirovski, Proximal method with rate of convergence $O(1 / t)$ for variational inequalities with Lipschitz continuous monotone operators and smooth convex-concave saddle point problems, SIAM J. Optim. 15, 229-251 (2004).

[14] A. Nemirovski, Interior point polynomial time methods in convex programming, Lecture Notes (Dept. of ISyE, Georgia Tech., GA, USA, 2004).

[15] A. Nemirovski, L. Tunçel, "Cone-free" primal-dual path-following and potential reduction polynomial time interior-point methods, Math. Prog. 102, 261-294 (2005).

[16] A. Nemirovski, D. B. Yudin, Problem Complexity and Method Efficiency in Optimization, Wiley-Interscience Series in Discrete Mathematics (John Wiley \& Sons, Inc., New York, 1983).

[17] Yu. Nesterov, Unconstrained convex minimization in relative scale, Math. Oper. Res. 34, 180-193 (2009).

[18] Yu. Nesterov, Rounding of convex sets and efficient gradient methods for linear programming problems, Optim. Methods Softw. 23, 109-128 (2008).

[19] Yu. Nesterov, Dual extrapolation and its applications for solving variational inequalities and related problems, Math. Prog. 109, 319-344 (2007).

[20] Yu. Nesterov, Smooth minimization of nonsmooth functions, Math. Prog. 103, 127-152 (2005).

[21] Yu. Nesterov, A. Nemirovskii, Interior Point Polynomial Methods in Convex Programming (SIAM Series in Applied Mathematics, SIAM: Philadelphia, 1994).

[22] S. A. Plotkin, D. B. Shmoys, É. Tardos, Fast approximation algorithms for fractional packing and covering problems, Math. Oper. Res. 20, 257-301 (1995).

[23] J. Renegar, Hyperbolic programs, and their derivative relaxations, Found. Comput. Math. 6, 59-79 (2006).

[24] F. Shahrokhi, D. W. Matula, The maximum concurrent flow problem, J. Assoc. Comput. Mach. 37, 318-334 (1990).

\section{APPENDIX}

In the following two proofs we work out the derivatives and their estimates in detail. Even though these two propositions admit shorter proofs (along the same lines), we include the detailed computations below partly for the hope that they will be useful for future research.

Proposition A.1 Let $p,|p| \geq 2$, be integer. Consider the following functions of $y \in \mathbb{S}_{++}^{d}$ :

$$
F(y):=\operatorname{Tr}\left(y^{p}\right), f(y):=(F(y))^{1 / p} .
$$

Then $f$ is convex when $p \geq 2, f$ is concave when $p \leq-2$, and

$$
y \succ 0, y \pm h \succeq 0 \Rightarrow\left|D^{3} f(y)[h, h, h]\right| \leq O(1)|p|\left|D^{2} f(y)[h, h]\right| .
$$


Proof. $\mathbf{1}^{0}$. Let us compute the derivatives of $F$ and $f$. We have

$$
\begin{gathered}
D f(y)[h]=\frac{1}{p}(F(y))^{1 / p} \frac{D F(y)[h]}{F(y)}=f(y) \frac{D F(y)[h]}{p F(y)} \\
D^{2} f(y)[h, h]=f(y)\left(\frac{D F(y)[h]}{p F(y)}\right)^{2}+f(y) \frac{D^{2} F(y)[h, h]}{p F(y)}-f(y) \frac{(D F(y)[h])^{2}}{p F^{2}(y)} \\
=\quad f(y)\left[(1-p)\left(\frac{D F(y)[h]}{p F(y)}\right)^{2}+\frac{D^{2} F(y)[h, h]}{p F(y)}\right] \\
\quad D^{3} f(y)[h, h, h] \\
=\quad f(y)\left[(1-p)\left(\frac{D F(y)[h]}{p F(y)}\right)^{3}+\frac{D F(y)[h] D^{2} F(y)[h, h]}{p^{2} F^{2}(y)}\right] \\
+f(y)\left[2(1-p) \frac{D F(y)[h]}{p^{2} F(y)}\left[\frac{D^{2} F(y)[h, h]}{F(y)}-\frac{(D F(y)[h])^{2}}{F^{2}(y)}\right]-\frac{D F(y)[h] D^{2} F(y)[h, h]}{p F^{2}(y)}+\frac{D^{3} F(y)[h, h, h]}{p F(y)}\right] \\
=\quad f(y)\left[(2 p-1)(p-1)\left(\frac{D F(y)[h]}{p F(y)}\right)^{3}-3(p-1) \frac{D F(y)[h] D^{2} F(y)[h, h]}{p^{2} F^{2}(y)}+\frac{D^{3} F(y)[h, h, h]}{p F(y)}\right] .
\end{gathered}
$$

Let $\left\{e_{j}\right\}$ be the orthonormal eigenbasis of $y$ and let $y_{j}$ denote the corresponding eigenvalues of $y$ (i.e., $y e_{j}=y_{j} e_{j}$ ). Further let $\bar{h}_{k j}=e_{j}^{T} h e_{k}, \eta_{k j}=y_{k}^{-1 / 2} y_{j}^{-1 / 2} \bar{h}_{k j}$. We have, assuming all $y_{j}$ 's distinct:

$$
\begin{aligned}
D F(y)[h] & =p \operatorname{Tr}\left(y^{p-1} h\right)=p \sum_{j} y_{j}^{p-1} \bar{h}_{j j}=\frac{1}{2 \pi i} \oint p \sum_{j} \frac{\bar{h}_{j j} z^{p-1}}{z-y_{j}} d z=\frac{1}{2 \pi i} \oint p \operatorname{Tr}\left((z I-y)^{-1} h\right) z^{p-1} d z \\
D^{2} F(y)[h, h] & =\frac{1}{2 \pi i} \oint p \operatorname{Tr}\left((z I-y)^{-1} h(z I-y)^{-1} h\right) z^{p-1} d z=\frac{1}{2 \pi i} \oint p \sum_{j, k} \frac{\bar{h}_{j k}^{2} z^{p-1}}{\left(z-y_{j}\right)\left(z-y_{k}\right)} d z \\
& =\sum_{j \neq k} \bar{h}_{j k}^{2} p \frac{y_{j}^{p-1}-y_{k}^{p-1}}{y_{j}-y_{k}}+\sum_{j} p(p-1) \bar{h}_{j j}^{2} y_{j}^{p-2} \\
D^{3} F(y)[h, h, h] & =\frac{2}{2 \pi i} \oint p \operatorname{Tr}\left((z I-y)^{-1} h(z I-y)^{-1} h(z I-y)^{-1} h\right) z^{p-1} d z \\
& =\frac{2}{2 \pi i} \oint p \sum_{j, k, \ell} \frac{\bar{h}_{j k} \bar{h}_{k \ell} \bar{h}_{\ell j} z^{p-1}}{\left(z-y_{j}\right)\left(z-y_{k}\right)\left(z-y_{l}\right)} d z=2 p \sum_{j, k, \ell} \bar{h}_{j k} \bar{h}_{k \ell} \bar{h}_{\ell j} \Gamma\left(y_{j}, y_{k}, y_{\ell}\right)
\end{aligned}
$$

where, for distinct $a, b, c$,

$$
\Gamma(a, b, c)=\frac{a^{p-1}}{(a-b)(a-c)}+\frac{b^{p-1}}{(b-a)(b-c)}+\frac{c^{p-1}}{(c-a)(c-b)},
$$

and

$$
\Gamma(a, b, c)=\lim _{\substack{\left(a^{\prime}, b^{\prime}, c^{\prime}\right) \rightarrow(a, b, c) \\ a^{\prime} \neq b^{\prime} \neq c^{\prime} \neq a^{\prime}}} \Gamma\left(a^{\prime}, b^{\prime}, c^{\prime}\right)
$$

Let $q=|p|$, and let $k \neq j$. When $p \geq 2$, we have $y_{j} y_{k} \frac{y_{j}^{p-1}-y_{k}^{p-1}}{y_{j}-y_{k}}=\sum_{\substack{\alpha+\beta=q \\ \alpha, \beta \geq 1}} y_{j}^{\alpha} y_{k}^{\beta}$. From now on, all the sums of the type $\sum_{\alpha+\beta=q}$ start from zero, unless we state otherwise. When $p \leq-2$, we have $\frac{y_{j}^{p-1}-y_{k}^{p-1}}{y_{j}-y_{k}}=$ $\frac{\left(1 / y_{j}\right)^{q+1}-\left(1 / y_{k}\right)^{q+1}}{y_{j} y_{k}\left(\left(1 / y_{k}\right)-\left(1 / y_{j}\right)\right)}=-y_{j}^{-1} y_{k}^{-1} \sum_{\alpha+\beta=q} y_{j}^{-\alpha} y_{k}^{-\beta}$, that is, $y_{j} y_{k} \frac{y_{j}^{p-1}-y_{k}^{p-1}}{y_{j}-y_{k}}=-\sum_{\alpha+\beta=q} y_{j}^{-\alpha} y_{k}^{-\beta}$. It follows that

$$
f^{-1}(y) D f(y)[h]=\frac{\sum_{j} y_{j}^{p} \eta_{j j}}{\sum_{j} y_{j}^{p}}=\sum_{j} p_{j} \eta_{j j}=: \mu ; \quad\left[p_{j}:=\frac{y_{j}^{p}}{\sum_{k} y_{k}^{p}}\right]
$$


further, in the case of $p \geq 2$ we have

$$
\begin{aligned}
& f^{-1}(y) D^{2} f(y)[h, h]=(1-p) \mu^{2}+\frac{p^{-1} D^{2} F(y)[h, h]}{F(y)}=(1-p) \mu^{2}+\frac{\sum_{j \neq k} \eta_{j k}^{2} \sum_{\substack{\alpha+\beta=p \\
\alpha, \beta \geq 1}} y_{j}^{\alpha} y_{k}^{\beta}+\sum_{j}(p-1) \eta_{j j}^{2} y_{j}^{p}}{\sum_{\ell} y_{\ell}^{p}} \\
& =(p-1)\left[\sum_{j} p_{j} \eta_{j j}^{2}-\mu^{2}\right]+\frac{\sum_{j \neq k} \eta_{j k}^{2} \sum_{\substack{\alpha+\beta=p \\
\alpha, \beta>1}} y_{j}^{\alpha} y_{k}^{\beta}}{\sum_{j \neq k} \eta_{j k}^{2} \sum_{\alpha+\beta=p}^{p} y_{j}^{\alpha} y_{k}^{\beta}}
\end{aligned}
$$

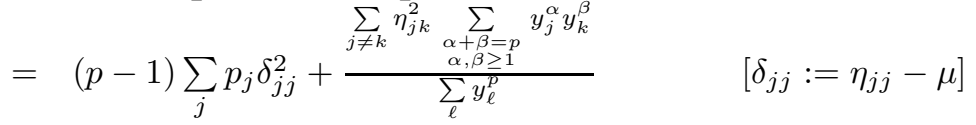

while in the case of $p \leq-2$ we have

$$
\begin{aligned}
f^{-1}(y) D^{2} f(y)[h, h] & =(1-p) \mu^{2}+\frac{p^{-1} D^{2} F(y)[h, h]}{F(y)}=(1-p) \mu^{2}+\frac{-\sum_{j \neq k} \eta_{j k}^{2} \sum_{\alpha+\beta=|p|} y_{j}^{-\alpha} y_{k}^{-\beta}+\sum_{j}(p-1) \eta_{j j}^{2} y_{j}^{p}}{\sum_{\ell} y_{\ell}^{p}} \\
& =(p-1)\left[\sum_{j} p_{j} \eta_{j j}^{2}-\mu^{2}\right]-\frac{\sum_{j \neq k} \eta_{j k}^{2} \sum_{\alpha+\beta=|p|} y_{j}^{-\alpha} y_{k}^{-\beta}}{\sum_{\ell} y_{\ell}^{p}} \\
& =(p-1) \sum_{j} p_{j} \delta_{j j}^{2}-\frac{\sum_{j \neq k} \eta_{j k}^{2} \sum_{\alpha+\beta=|p|} y_{j}^{-\alpha} y_{k}^{-\beta}}{\sum_{\ell} y_{\ell}^{p}} .
\end{aligned}
$$

Finally, in the case of $p \geq 2$ we have

$$
\begin{aligned}
f^{-1}(y) D^{3} f(y)[h, h, h]= & (2 p-1)(p-1) \mu^{3}-3(p-1) \mu\left[(p-1) \sum_{j} p_{j} \eta_{j j}^{2}+\frac{\sum_{j \neq k} \eta_{j k}^{2} y_{j} y_{k} \sum_{\alpha+\beta=p-2} y_{j}^{\alpha} y_{k}^{\beta}}{\sum_{\ell} y_{\ell}^{p}}\right] \\
& +\frac{2 \sum_{j, k, \ell} y_{j} y_{k} y_{\ell} \eta_{j k} \eta_{k \ell} \eta_{\ell j} \Gamma\left(y_{j}, y_{k}, y_{\ell}\right)}{\sum_{\nu} y_{\nu}^{p}}
\end{aligned}
$$

and for distinct positive $a, b, c$ the following relations hold:

$$
\begin{aligned}
\Gamma(a, b, c) & =\frac{a^{p-1}}{(a-b)(a-c)}+\frac{b^{p-1}}{(b-c)(b-a)}+\frac{c^{p-1}}{(c-a)(c-b)}=\frac{a^{p-1}-c^{p-1}}{(a-b)(a-c)}+\frac{b^{p-1}-c^{p-1}}{(b-c)(b-a)} \\
& =\sum_{\alpha+\beta=p-2}\left[\frac{a^{\alpha} c^{\beta}}{a-b}+\frac{b^{\alpha} c^{\beta}}{b-a}\right]=\sum_{0 \leq \beta \leq p-2} c^{\beta} \sum_{0 \leq \alpha \leq p-2-\beta} \frac{a^{\alpha}-b^{\alpha}}{a-b} \\
& =\sum_{\alpha+\beta+\gamma=p-3} a^{\alpha} b^{\beta} c^{\gamma} .
\end{aligned}
$$


This concluding identity clearly remains valid when not all $a, b, c$ are distinct. Thus, in the case of $p \geq 2$ we have

$$
\begin{aligned}
& f^{-1}(y) D^{3} f(y)[h, h, h] \\
& =(2 p-1)(p-1) \mu^{3}-3(p-1) \mu\left[(p-1) \mu^{2}+(p-1) \sum_{j} p_{j} \delta_{j j}^{2}+\frac{\sum_{j \neq k} \eta_{j k}^{2} y_{j} y_{k} \sum_{\alpha+\beta=p-2} y_{j}^{\alpha} y_{k}^{\beta}}{\sum_{\ell} y_{\ell}^{\rho}}\right] \\
& +\frac{2 \sum_{j, k, \ell} y_{j} y_{k} y_{\ell} \eta_{j k} \eta_{k \ell} \eta_{\ell j} \sum_{\alpha+\beta+\gamma=p-3} y_{j}^{\alpha} y_{k}^{\beta} y_{\ell}^{\gamma}}{\sum_{\nu} y_{\nu}^{p}} \\
& =\left[-p^{2}+3 p-2\right] \mu^{3}-3(p-1)^{2} \mu \sum_{j} p_{j} \delta_{j j}^{2}+2 \sum_{j} p_{j} \eta_{j j}^{3} \sum_{\alpha+\beta+\gamma=p-3} 1-3(p-1) \mu \frac{\sum_{\substack{j \neq k \\
\eta_{j k}^{2}}}^{\substack{\alpha+\beta=p \\
\alpha, \beta>1}} y_{j}^{\alpha} y_{k}^{\beta}}{\sum_{\ell} y_{\ell}^{p}} \\
& +\frac{2 \sum_{(j \neq k) \backslash(j \neq \ell)} \eta_{j k} \eta_{k \ell} \eta_{\ell j} \sum_{\substack{\alpha+\beta+\gamma=p \\
\alpha, \beta, \gamma \geq 1}} y_{j}^{\alpha} y_{k}^{\beta} y_{\ell}^{\gamma}}{\sum_{\nu} y_{\nu}^{\rho}} \\
& =\left[-p^{2}+3 p-2\right] \mu^{3}-3(p-1)^{2} \mu \sum_{j} p_{j} \delta_{j j}^{2}+(p-1)(p-2) \sum_{j} p_{j} \eta_{j j}^{3}-3(p-1) \mu \frac{\sum_{\substack{j \neq k \\
\eta_{j k}^{2}}}^{\sum_{\substack{\alpha+\beta=p \\
\alpha+\beta \geq 1}} y_{j}^{\alpha} y_{k}^{\beta}}}{\sum_{\ell} y_{\ell}^{\rho}} \\
& +\frac{2 \sum_{(j \neq k) \backslash(j \neq \ell)} \eta_{j k} \eta_{k \ell} \eta_{\ell j} \sum_{\substack{\alpha+\beta+\gamma=p \\
\alpha, \beta, \gamma \geq 1}} y_{j}^{\alpha} y_{k}^{\beta} y_{\ell}^{\gamma}}{\sum_{\nu} y_{\nu}^{\nu}} \\
& =\left[-p^{2}+3 p-2\right] \mu^{3}-3(p-1)^{2} \mu \sum_{j} p_{j} \delta_{j j}^{2}+(p-1)(p-2)\left[\sum_{j} p_{j}\left[\mu^{3}+3 \mu^{2} \delta_{j j}+3 \mu \delta_{j j}^{2}+\delta_{j j}^{3}\right]\right]
\end{aligned}
$$

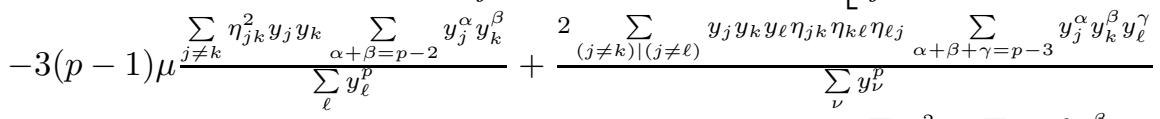

$$
\begin{aligned}
& =-3(p-1) \mu \sum_{j} p_{j} \delta_{j j}^{2}+(p-1)(p-2) \sum_{j} p_{j} \delta_{j j}^{3}-3(p-1) \mu \frac{\sum_{\substack{j \neq k \\
j k}}^{\substack{\alpha+\beta=p \\
\alpha, \beta>1}} y_{j}^{\alpha} y_{k}^{\beta}}{\sum_{\ell} y_{\ell}^{p}} \\
& +\frac{2 \sum_{(j \neq k) \backslash(j \neq \ell)} \eta_{j k} \eta_{k \ell} \eta_{\ell j} \sum_{\substack{\alpha+\beta+\gamma=p \\
\alpha, \beta, \gamma \geq 1}} y_{j}^{\alpha} y_{k}^{\beta} y_{\ell}^{\gamma}}{\sum_{\nu} y_{\nu}^{\nu}} \text {. }
\end{aligned}
$$

In the case of $p \leq-2$ we get $\Gamma(a, b, c)=\frac{1}{a b c} \sum_{\alpha+\beta+\gamma=|p|} a^{-\alpha} b^{-\beta} c^{-\gamma}$, and the resulting formula for $f^{-1}(y) D^{3} f(y)[h, h, h]$ becomes

$$
\begin{aligned}
f^{-1}(y) D^{3} f(y)[h, h, h]= & -3(p-1) \mu \sum_{j} p_{j} \delta_{j j}^{2}+(p-1)(p-2) \sum_{j} p_{j} \delta_{j j}^{3}+3(p-1) \mu \frac{\sum_{j \neq k} \eta_{j k}^{2} \sum_{\alpha+\beta=|p|} y_{j}^{-\alpha} y_{k}^{-\beta}}{\sum_{\ell}^{p} y_{\ell}^{p}} \\
& +\frac{2 \sum_{(j \neq k) \mid(j \neq \ell)} \eta_{j k} \eta_{k \ell} \eta_{\ell j} \sum_{\alpha+\beta+\gamma=|p|} y_{j}^{-\alpha} y_{k}^{-\beta} y_{\ell}^{-\gamma}}{\sum_{\nu} y_{\nu}^{\rho}} .
\end{aligned}
$$

The resulting formulas, obtained for the case when all $y_{j}$ are distinct, clearly remain valid for all $y \succ 0$. Thus, for all $y \succ 0$ and all $h$ we have, setting $f=f(y), d f=D f(y)[h], d^{2} f=D^{2} f(y)[h, h]$, $d^{3} f=D^{3} f(y)[h, h, h]$ : 


$$
\begin{aligned}
& f^{-1} d f=\sum_{j} p_{j} \eta_{j j}=: \mu \\
& \underline{p \geq 2:} \\
& \begin{array}{l}
f^{-1} d^{2} f=(p-1) \sum_{j} p_{j} \delta_{j j}^{2}+\frac{\sum_{j \neq k} \eta_{j k}^{2} \sum_{\substack{\alpha+\beta=p \\
\alpha, \beta \geq 1}} y_{j}^{\alpha} y_{k}^{\beta}}{\sum_{\nu} y_{\nu}^{p}} \\
\underline{p \leq-2:} \\
f^{-1} d^{2} f=(p-1) \sum_{j} p_{j} \delta_{j j}^{2}-\frac{\sum_{j \neq k} \eta_{j k}^{2} \sum_{\alpha+\beta=|p|} y_{j}^{-\alpha} y_{k}^{-\beta}}{\sum_{\nu} y_{\nu}^{p}}
\end{array} \\
& \underline{p \geq 2:} \\
& f^{-1} d^{3} f=-3(p-1) \mu \sum_{j} p_{j} \delta_{j j}^{2}+(p-1)(p-2) \sum_{j} p_{j} \delta_{j j}^{3} \\
& -3(p-1) \mu \frac{\sum_{j \neq k} \eta_{j k}^{2} \sum_{\substack{\alpha+\beta=p \\
\alpha, \beta \geq 1}} y_{j}^{\alpha} y_{k}^{\beta}}{\sum_{\nu} y_{\nu}^{p}}+2 \frac{\sum_{(j \neq k) \mid(j \neq \ell)} \eta_{j k} \eta_{k \ell} \eta_{\ell j} \sum_{\substack{\alpha+\beta+\gamma=p \\
\alpha, \beta, \gamma \geq 1}} y_{j}^{\alpha} y_{k}^{\beta} y_{\ell}^{\gamma}}{\sum_{\nu} y_{\nu}^{p}} \\
& \underline{p \leq-2:} \\
& \begin{array}{l}
f^{-1} d^{3} f=-3(p-1) \mu \sum_{j} p_{j} \delta_{j j}^{2}+(p-1)(p-2) \sum_{j} p_{j} \delta_{j j}^{3} \\
\quad+3(p-1) \mu \frac{\sum_{j \neq k} \eta_{j k}^{2} \sum_{\alpha+\beta=|p|} y_{j}^{-\alpha} y_{k}^{-\beta}}{\sum_{\nu} y_{\nu}^{p}}+2 \frac{\sum_{(j \neq k) \mid(j \neq \ell)} \eta_{j k} \eta_{k \ell} \eta_{\ell j} \sum_{\alpha+\beta+\gamma=|p|} y_{j}^{-\alpha} y_{k}^{-\beta} y_{\ell}^{-\gamma}}{\sum_{\nu} y_{\nu}^{p}},
\end{array}
\end{aligned}
$$$$
\left[p_{j}=\frac{y_{j}^{p}}{\sum_{k} y_{k}^{p}}\right]
$$$$
\left[\delta_{j j}=\eta_{j j}-\mu\right]
$$

where $y_{j}$ are the eigenvalues of $y, \eta_{i j}=y_{i}^{-1 / 2}\left(e_{i}^{T} h e_{j}\right) y_{j}^{-1 / 2}$ and $e_{i}$ form an orthonormal eigenbasis of $y$. Note that under the premise of (25) we have

$$
-I \preceq \eta \preceq I .
$$

$\mathbf{2}^{0}$. In the sequel, we focus on the case of $p \geq 2$. The reasoning in the case of $p \leq-2$ is similar.

We have the following

Lemma A.1 Suppose $y \succ 0$ and that (27) holds. Then

$$
\begin{aligned}
& \text { (a) }\left|\delta_{j j}\right| \leq 2 \\
& \text { (b) }|\mu| \leq 1 \\
& \text { (c) }\left|\sum_{\mid j \neq k) \mid(j \neq \ell)} \eta_{j k} \eta_{k \ell} \eta_{\ell j} \sum_{\substack{\alpha+\beta+\gamma=p \\
\alpha, \beta, \gamma \geq 1}} y_{j}^{\alpha} y_{k}^{\beta} y_{\ell}^{\gamma}\right| \leq O(1) p \underbrace{\sum_{j \neq k} \eta_{j k}^{2} \sum_{\substack{\alpha+\beta=p \\
\alpha, \beta \geq 1}}^{\sum_{j}} y_{j}^{\alpha} y_{k}^{\beta}}_{\mathcal{R}} .
\end{aligned}
$$

Proof. By (27), we have $\left|\eta_{j j}\right| \leq 1$. Since $\mu$ is a convex combination of $\eta_{j j}$ and $\delta_{j j}=\eta_{j j}-\mu,(a),(b)$ follow.

Let $\zeta$ be the matrix obtained from $\eta$ by replacing the diagonal entries with 0 . By (27), we have

$$
-2 I \preceq \zeta \preceq 2 I .
$$


We now have

$$
\sum_{(j \neq k) \mid(j \neq \ell)} \eta_{j k} \eta_{k \ell} \eta_{\ell j} \sum_{\substack{\alpha+\beta+\gamma=p \\ \alpha, \beta, \gamma \geq 1}} y_{j}^{\alpha} y_{k}^{\beta} y_{\ell}^{\gamma}=3 \underbrace{\sum_{k \neq j} \eta_{j j} \eta_{j k}^{2} \sum_{\substack{\alpha+\beta+\gamma=p \\ \alpha, \beta, \gamma \geq 1}} y_{j}^{\alpha+\beta} y_{k}^{\gamma}}_{I_{1}}+\underbrace{\sum_{\substack{\alpha+\beta+\gamma=p \\ \alpha+\beta, \gamma \geq 1}} \overbrace{\sum_{j, k, \ell} \zeta_{j k} \zeta_{k \ell} \zeta_{\ell j} y_{j}^{\alpha} y_{k}^{\beta} y_{\ell}^{\gamma}}^{\Psi(\alpha, \beta, \gamma)}}_{I_{2}} .
$$

We also have

$$
\left|I_{1}\right| \leq \sum_{k \neq j}\left|\eta_{j j}\right| \eta_{j k}^{2} \sum_{\substack{\alpha+\beta+\gamma=p \\ \alpha, \beta, \gamma \geq 1}} y_{j}^{\alpha+\beta} y_{k}^{\gamma} \leq \sum_{\substack{\alpha+\beta+\gamma=p \\ \alpha, \beta, \gamma \geq 1}} \sum_{j \neq k} \eta_{j k}^{2} y_{j}^{\alpha+\beta} y_{k}^{\gamma} \leq(p-2) \sum_{1 \leq \gamma<p} \sum_{j \neq k} \eta_{j k}^{2} y_{j}^{p-\gamma} y_{k}^{\gamma},
$$

where the second inequality is given by (27). Further, $\Psi(\alpha, \beta, \gamma)$ clearly is symmetric in the arguments, which gives the first inequality in the following chain (where $Y=\operatorname{Diag}\left\{y_{1}, \ldots, y_{n}\right\}$ and $\|z\|_{F}$ is the Frobenius norm of a matrix):

$$
\begin{aligned}
& \left|I_{2}\right| \leq \sum_{\substack{\alpha+\beta+\gamma=p \\
1 \leq \alpha \leq \beta>\gamma \geq 1}} 6\left|\sum_{j, k, \ell} \zeta_{j k} \zeta_{k \ell} \zeta_{\ell j} y_{j}^{\alpha} y_{k}^{\frac{p-2 \alpha}{2}} y_{k}^{\frac{p-2 \gamma}{2}} y_{\ell}^{\gamma}\right| \\
& \leq 6 \sum_{1 \leq \alpha, \gamma<p / 2}\left|\operatorname{Tr}\left(\left[Y^{\alpha} \zeta Y^{\frac{p-2 \alpha}{2}}\right]\left[Y^{\frac{p-2 \gamma}{2}} \zeta Y^{\gamma}\right] \zeta\right)\right| \\
& \leq 12 \sum_{1 \leq \alpha, \gamma<p / 2} \underbrace{\left\|Y^{\alpha} \zeta Y^{\frac{p-2 \alpha}{2}}\right\|_{F}}_{\sqrt{S_{\alpha}}}\left\|Y^{\frac{p-2 \gamma}{2}} \zeta Y^{\gamma}\right\|_{F} \\
& =12 \sum_{1 \leq \alpha, \gamma<p / 2} \sqrt{S_{\alpha}} \sqrt{S_{\gamma}} \\
& =12\left(\sum_{1 \leq \alpha<p / 2} S_{\alpha}^{1 / 2}\right)^{2} \leq 6 p \sum_{1 \leq \alpha<p / 2} S_{\alpha} \leq 6 p \mathcal{R} \text {. } \\
& {\left[S_{\alpha}=\sum_{\mu, \nu} y_{\mu}^{2 \alpha} \zeta_{\mu \nu}^{2} y_{\nu}^{p-2 \alpha} \Rightarrow \sum_{1 \leq \alpha<p / 2} S_{\alpha} \leq \mathcal{R}\right]}
\end{aligned}
$$

Combining (31), (32), we arrive at (28.c).

$\mathbf{3}^{0}$. Combining (28) with (26), we arrive at the desired inequality (25).

Proposition A.2 Let

$$
F(y):=\operatorname{Tr}(\exp \{y\}), f(y):=\ln F(y) \quad\left[y \in \mathbb{S}^{d}\right] .
$$

Then

$$
(L I \succeq y \succ 0,-y \preceq h \preceq y) \Rightarrow\left|D^{3} f(y)[h, h, h]\right| \leq O(1) L D^{2} f(y)[h, h] .
$$

Proof. $\mathbf{1}^{0}$. Let us compute the derivatives of $f(y)$ assuming $y \succ 0$.

We have

$$
\begin{aligned}
D f(y)[h] & =\frac{D F(y)[h]}{F(y)}=\frac{\operatorname{Tr}(\exp \{y\} h)}{\operatorname{Tr}(\exp \{y\})} \\
D^{2} f(y)[h, h] & =-\left(\frac{D F(y)[h]}{F(y)}\right)^{2}+\frac{D^{2} F(y)[h, h]}{F(y)} \\
D^{3} f(y)[h, h, h] & =2\left(\frac{D F(y)[h]}{F(y)}\right)^{3}-3 \frac{D F(y)[h] D^{2} F(y)[h, h]}{F^{2}(y)}+\frac{D^{3} F(y)[h, h]}{F(y)} .
\end{aligned}
$$


Let $y \succ 0$, let $\left\{e_{j}\right\}$ be the orthonormal eigenbasis of $y$ and let $y_{j}$ be the corresponding eigenvalues as before. Also let $\bar{h}_{k j}=e_{j}^{T} h e_{k}$, and finally let $\eta_{k j}=y_{k}^{-1 / 2} y_{j}^{-1 / 2} \bar{h}_{k j}$. We have, assuming all $y_{j}$ 's are distinct:

$$
\begin{aligned}
D F(y)[h] & =\operatorname{Tr}(\exp \{y\} h)=\frac{1}{2 \pi i} \oint \operatorname{Tr}\left((z I-y)^{-1} h\right) \exp \{z\} d z=\sum_{j} \exp \left\{y_{j}\right\} \bar{h}_{j j} \\
& =\sum_{j} \eta_{j j}^{2} y_{j} \exp \left\{y_{j}\right\} \\
D^{2} F(y)[h, h] & =\frac{1}{2 \pi i} \oint \operatorname{Tr}\left((z I-y)^{-1} h(z I-y)^{-1} h\right) \exp \{z\} d z=\frac{1}{2 \pi i} \oint \sum_{j, k} \frac{\bar{h}_{j k}^{2} \exp \{z\}}{\left(z-y_{j}\right)\left(z-y_{k}\right)} d z \\
& =\sum_{j \neq k} \bar{h}_{j k}^{2} \frac{\exp \left\{y_{j}\right\}-\exp \left\{y_{k}\right\}}{y_{j}-y_{k}}+\sum_{j} \bar{h}_{j j}^{2} \exp \left\{y_{j}\right\} \\
& =\sum_{p=1}^{\infty} \sum_{j \neq k} \bar{h}_{j k}^{2} \frac{1}{p_{1}} \sum_{\alpha+\beta=p-1} y_{j}^{\alpha} y_{k}^{\beta}+\sum_{j} \bar{h}_{j j}^{2} \exp \left\{y_{j}\right\} \\
& =\sum_{p=1}^{\infty} \sum_{j \neq k} \sum_{\alpha+\beta=p+1} \eta_{j k}^{2} \frac{1}{p !} y_{j}^{\alpha} y_{k}^{\beta}+\sum_{j} \eta_{j j}^{2} y_{j}^{2} \exp \left\{y_{j}\right\} \\
D^{3} F(y)[h, h, h] & \left.=\frac{2}{2 \pi i} \oint \operatorname{Tr}((z I-y))^{-1} h(z I-y)^{-1} h(z I-y)^{-1} h\right) \exp \{z\} d z \\
& =\frac{2}{2 \pi i} \oint \sum_{j, k, \ell} \frac{\bar{h}_{j k} \bar{h}_{k \ell} \bar{h}_{\ell j}\left(z-y_{j}\right)\left(z-y_{k}\right)\left(z-y_{l}\right)}{\left(z-y_{l}\right)} d z \\
& =2 \sum_{j, k, \ell} \bar{h}_{j k} \bar{h}_{k \ell} \bar{h}_{\ell j} \Gamma\left(y_{j}, y_{k}, y_{\ell}\right)
\end{aligned}
$$

where

$$
\Gamma(a, b, c)=\frac{\exp \{a\}}{(a-b)(a-c)}+\frac{\exp \{b\}}{(b-a)(b-c)}+\frac{\exp \{c\}}{(c-a)(c-b)}
$$

for distinct $a, b, c$, and

$$
\Gamma(a, b, c)=\lim _{\substack{\left(a^{\prime}, b^{\prime}, c^{\prime}\right) \rightarrow(a, b, c) \\ a^{\prime} \neq b^{\prime} \neq c^{\prime} \neq a^{\prime}}} \Gamma\left(a^{\prime}, b^{\prime}, c^{\prime}\right)
$$

Assuming $a, b, c$ distinct, we have

$$
\begin{aligned}
\Gamma(a, b, c) & =\frac{\exp \{a\}}{(a-b)(a-c)}+\frac{\exp \{b\}}{(b-a)(b-c)}+\frac{\exp \{c\}}{(c-a)(c-b)}=\frac{\exp \{a\}-\exp \{c\}}{(a-b)(a-c)}+\frac{\exp \{b\}-\exp \{c\}}{(b-a)(b-c)} \\
& =\frac{1}{a-b}\left[\sum_{p=1}^{\infty} \frac{1}{p !} \sum_{\alpha+\beta=p-1} a^{\alpha} b^{\beta}-\sum_{p=1}^{\infty} \frac{1}{p !} \sum_{\alpha+\beta=p-1} c^{\alpha} b^{\beta}\right] \\
& =\sum_{p=2}^{\infty} \frac{1}{p !} \sum_{\substack{\alpha+\beta=p-1 \\
\alpha \geq 1}} b^{\beta} \frac{a^{\alpha}-c^{\alpha}}{a-c}=\sum_{p=2}^{\infty} \frac{1}{p !} \sum_{\mu+\nu+\beta=p-2} a^{\mu} c^{\nu} b^{\beta} .
\end{aligned}
$$

The resulting representation is, of course, valid for all $a, b, c$. We therefore get

$$
\begin{aligned}
D^{3} F(y)[h, h, h] & =2 \sum_{j, k, \ell} \bar{h}_{j k} \bar{h}_{k \ell} \bar{h}_{\ell j} \sum_{p=2}^{\infty} \frac{1}{p !} \sum_{\alpha+\beta+\gamma=p-2} y_{j}^{\alpha} y_{k}^{\beta} y_{\ell}^{\gamma} \\
& =2 \sum_{p=2}^{\infty} \sum_{j, k, \ell} \sum_{\substack{\alpha+\beta+\gamma=p+1 \\
\alpha, \beta, \gamma \geq 1}} \eta_{j k} \eta_{k \ell} \eta_{\ell j} \frac{1}{p !} y_{j}^{\alpha} y_{k}^{\beta} y_{\ell}^{\gamma} .
\end{aligned}
$$

We now have

$$
\begin{aligned}
& D f(y)[h]=\frac{\sum_{j} \exp \left\{y_{j}\right\} \eta_{j j} y_{j}}{\sum_{j} \exp \left\{y_{j}\right\}}=\sum_{j} p_{j}\left(y_{j} \eta_{j j}\right)=: \mu \quad\left[p_{j}:=\frac{\exp \left\{y_{j}\right\}}{\sum_{k} \exp \left\{y_{k}\right\}}\right], \\
& D^{2} f(y)[h, h]=-\left(\sum_{j} p_{j} y_{j} \eta_{j j}\right)^{2}+\frac{\sum_{p=1}^{\infty} \sum_{j \neq k} \sum_{\alpha+\beta=p+1} \eta_{j k}^{2} \frac{1}{p ! \beta} y_{j}^{\alpha} y_{k}^{\beta}+\sum_{j} y_{j}^{2} \eta_{j j}^{2} \exp \left\{y_{j}\right\}}{\sum_{j} \exp \left\{y_{j}\right\}} \\
& =\sum_{j} p_{j} \sigma_{j}^{2}+\frac{\sum_{p=1}^{\infty} \sum_{j \neq k} \sum_{\substack{\alpha=p+1 \\
\alpha, \beta \geq 1}} \eta_{j k}^{2} \frac{1}{p !} y_{j}^{\alpha} y_{k}^{\beta}}{\sum_{j} \exp \left\{y_{j}\right\}} \\
& {\left[\sigma_{j}:=y_{j} \eta_{j j}-\mu\right]}
\end{aligned}
$$


and

$$
\begin{aligned}
& D^{3} f(y)[h, h, h] \\
& =2 \mu^{3}-3 \mu\left(\frac{\sum_{p=1}^{\infty} \sum_{j \neq k} \sum_{\substack{\alpha \neq \beta=p+1 \\
\alpha, \beta>1}} \eta_{j k}^{2} \frac{1}{p !} y_{j}^{\alpha} y_{k}^{\beta}}{\sum_{j} \exp \left\{y_{j}\right\}}+\sum_{j} p_{j} y_{j}^{2} \eta_{j j}^{2}\right)+\frac{D^{3} F(y)[h, h, h]}{F} \\
& =2 \mu^{3}-3 \mu\left(\frac{\sum_{p=1}^{\infty} \sum_{j \neq k} \sum_{\substack{\alpha+\beta=p+1 \\
\alpha, \beta \geq 1}} \eta_{j k}^{2} \frac{1}{p !} y_{j}^{\alpha} y_{k}^{\beta}}{\sum_{\nu} \exp \left\{y_{\nu}\right\}}+\mu^{2}+\sum_{j} p_{j} \sigma_{j}^{2}\right)+\frac{2 \sum_{p=2}^{\infty} \sum_{j, k, \ell} \sum_{\substack{\alpha+\beta, \gamma=p+1 \\
\alpha, \beta, \gamma \geq 1}} \eta_{j k} \eta_{k \ell} \eta_{\ell j} \frac{1}{p !} y_{j}^{\alpha} y_{k}^{\beta} y_{\ell}^{\gamma}}{\sum_{\nu} \exp \left\{y_{\nu}\right\}} \\
& =-\mu^{3}-3 \mu \sum_{j} p_{j} \sigma_{j}^{2}-3 \mu \frac{\sum_{p=1}^{\infty} \sum_{j \neq k} \sum_{\substack{\alpha+\beta=p+1 \\
\alpha, \beta \geq 1}} \eta_{j k}^{2} \frac{1}{p !} y_{j}^{\alpha} y_{k}^{\beta}}{\sum_{\nu} \exp \left\{y_{\nu}\right\}}+\frac{2 \sum_{p=2}^{\infty} \frac{1}{p !} \sum_{j} \eta_{j j}^{3} \sum_{\substack{\alpha+\beta+\gamma=p+1 \\
\alpha, \beta}, \gamma \geq 1} y_{j}^{\alpha} y_{j}^{\beta} y_{j}^{\gamma}}{\sum_{\nu} \exp \left\{y_{\nu}\right\}} \\
& +\frac{2 \sum_{p=2}^{\infty} \sum_{\substack{j, k, \ell \\
(j \neq k) \mid(j \neq \ell)}} \sum_{\substack{\alpha+\beta+\gamma=p+1 \\
\alpha, \beta, \gamma \geq 1}} \eta_{j k} \eta_{k \ell} \eta_{\ell j} \frac{1}{p !} y_{j}^{\alpha} y_{k}^{\beta} y_{\ell}^{\gamma}}{\sum_{\nu} \exp \left\{y_{\nu}\right\}}
\end{aligned}
$$

Whence

$$
\begin{aligned}
& D^{3} f(y)[h, h, h] \\
& =-\mu^{3}-3 \mu \sum_{j} p_{j} \sigma_{j}^{2}-3 \mu \frac{\sum_{p=1}^{\infty} \sum_{j \neq k} \sum_{\alpha+\beta, p+1} \eta_{j k}^{2} \frac{1}{p !} y_{j}^{\alpha} y_{k}^{\beta}}{\sum_{\nu} \exp \left\{y_{\nu}\right\}}+\frac{2 \sum_{p=2}^{\infty} \frac{1}{p !} \sum_{j} \eta_{j j}^{3} y_{j}^{p+1} \frac{p(p-1)}{2}}{\sum_{\nu} \exp \left\{y_{\nu}\right\}} \\
& +\frac{2 \sum_{p=2}^{\infty} \sum_{\substack{j, k, \ell \\
(j \neq k) \mid(j \neq \ell)}} \sum_{\substack{\alpha+\beta+\gamma=p+1 \\
\alpha, \beta, \gamma \geq 1}} \eta_{j k} \eta_{k \ell} \eta_{\ell j} \frac{1}{p !} y_{j}^{\alpha} y_{k}^{\beta} y_{\ell}^{\gamma}}{\sum_{\nu} \exp \left\{y_{\nu}\right\}} \\
& =-\mu^{3}-3 \mu \sum_{j} p_{j} \sigma_{j}^{2}-3 \mu \frac{\sum_{p=1}^{\infty} \sum_{j \neq k} \sum_{\substack{\alpha=p+1 \\
\alpha, \beta>1}} \eta_{j k}^{2} \frac{1}{p !} y_{j}^{\alpha} y_{k}^{\beta}}{\sum_{\nu} \exp \left\{y_{\nu}\right\}}+\sum_{j} p_{j} \underbrace{\eta_{j j}^{3} y_{j}^{3}}_{\left(\sigma_{j}+\mu\right)^{3}} \\
& =\sum_{j} p_{j} \sigma_{j}^{3}-3 \mu \frac{\sum_{p=1}^{\infty} \sum_{j \neq k} \sum_{\substack{\alpha+\beta=p+1 \\
\alpha, \beta \geq 1}} \eta_{j k}^{2} \frac{1}{p !} y_{j}^{\alpha} y_{k}^{\beta}}{\sum_{\nu} \exp \left\{y_{\nu}\right\}}+\frac{2 \sum_{p=2}^{\infty} \sum_{\substack{j, k, \ell \\
(j \neq k) \backslash(j \neq \ell)}} \sum_{\substack{\alpha+\beta+\gamma=p+1 \\
\alpha, \beta, \gamma \geq 1}} \eta_{j k} \eta_{k \ell} \eta_{\ell j} \frac{1}{p !} y_{j}^{\alpha} y_{k}^{\beta} y_{\ell}^{\gamma}}{\sum_{\nu} \exp \left\{y_{\nu}\right\}} \\
& =\sum_{j} p_{j} \sigma_{j}^{3}-3 \mu \frac{\sum_{p=1}^{\infty} \sum_{j \neq k} \sum_{\substack{\alpha=p+1 \\
\alpha, \beta \geq 1}} \eta_{j k}^{2} \frac{1}{p !} y_{j}^{\alpha} y_{k}^{\beta}}{\sum_{\nu} \exp \left\{y_{\nu}\right\}}+\frac{6 \sum_{p=2}^{\infty} \sum_{j \neq \ell} \sum_{\substack{\alpha+\beta=p+1 \\
\alpha \geq 2, \beta \geq 1}} \eta_{j j} \eta_{j \ell}^{2} \frac{1}{p !}(\alpha-1) y_{j}^{\alpha} y_{\ell}^{\beta}}{\sum_{\nu} \exp \left\{y_{\nu}\right\}} \\
& +\frac{2 \sum_{p=2}^{\infty} \sum_{j \neq k \neq \ell \neq j} \sum_{\substack{\alpha+\beta+\gamma=p+1 \\
\alpha, \beta, \gamma \geq 1}} \eta_{j k} \eta_{k \ell} \eta_{\ell j} \frac{1}{p !} y_{j}^{\alpha} y_{k}^{\beta} y_{\ell}^{\gamma}}{\sum_{\nu} \exp \left\{y_{\nu}\right\}} .
\end{aligned}
$$


Thus,

$$
\begin{aligned}
& d f:=D f(y)[h]=\sum_{j} p_{j}\left(y_{j} \eta_{j j}\right)=\mu \\
& d^{2} f:=D^{2} f(y)[h, h]=\sum_{j} p_{j} \sigma_{j}^{2}+\left[\frac{\sum_{p=1}^{\infty} \sum_{\substack { j \neq k \\
\begin{subarray}{c}{\alpha+\beta=p+1 \\
\alpha, \beta \geq 1{ j \neq k \\
\begin{subarray} { c } { \alpha + \beta = p + 1 \\
\alpha , \beta \geq 1 } }\end{subarray}} \eta_{j k}^{2} \frac{1}{p !} y_{j}^{\alpha} y_{k}^{\beta}}{\sum_{j} \exp \left\{y_{j}\right\}}\right] \quad\left[\sigma_{j}=y_{j} \eta_{j j}-\mu\right]
\end{aligned}
$$

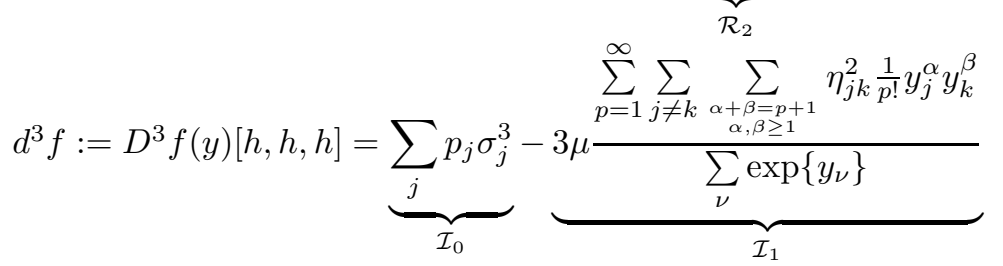

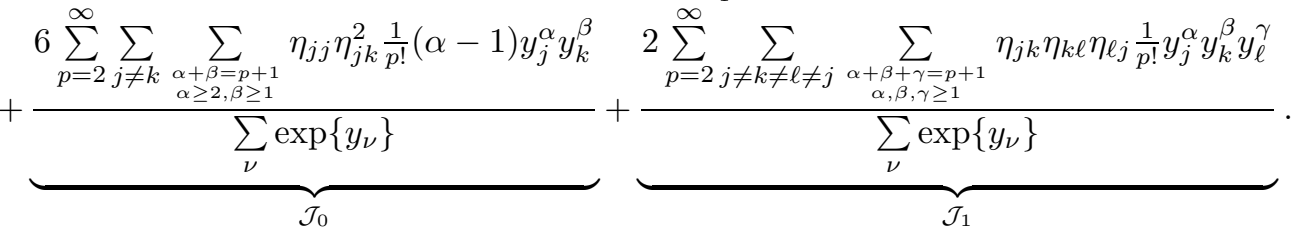

$$
\begin{aligned}
& {\left[p_{j}=\frac{\exp \left\{y_{j}\right\}}{\sum_{k} \exp \left\{y_{k}\right\}}\right]}
\end{aligned}
$$

The resulting formulas for the derivatives, although established under the assumption that all $y_{j}$ are distinct, clearly remain valid for all $y \succ 0$.

$\mathbf{2}^{0}$. Now let $y, h$ satisfy the premise of (33). Then

$$
0<y_{j} \leq L, j \in\{1,2, \ldots, n\}, \&-I \preceq \eta \preceq I .
$$

Whence

$$
|\mu| \leq L,\left|\sigma_{j}\right| \leq 2 L
$$

It follows that

$$
\left|\mathcal{I}_{0}\right|+\left|\mathcal{I}_{1}\right| \leq 3 L d^{2} f
$$

Further, we have

$$
\begin{aligned}
& \left|\mathcal{J}_{0}\right| \leq \frac{6 \sum_{p=2}^{\infty} \sum_{j \neq k} \sum_{\substack{\alpha+\beta=p+1 \\
\alpha \geq 2, \beta \geq 1}}\left|\eta_{j j}\right| \eta_{j k}^{2} \frac{1}{p !}(\alpha-1) y_{j}^{\alpha} y_{k}^{\beta}}{\sum_{\nu} \exp \left\{y_{\nu}\right\}} \\
& \leq \frac{6 \sum_{p=2}^{\infty} \sum_{j \neq k} \sum_{\substack{\alpha+\beta=p+1 \\
\alpha \geq 2, \beta \geq 1}} \eta_{j k}^{2} \frac{\alpha-1}{p !} y_{j}^{\alpha} y_{k}^{\beta}}{\sum_{\nu} \exp \left\{y_{\nu}\right\}} \\
& 6 \sum_{q=1}^{\infty} \sum_{j \neq k} \sum_{\alpha^{\prime}+\beta=q+1} \eta_{j k}^{2} y_{j} \frac{\alpha^{\prime}}{(q+1) !} y_{j}^{\alpha^{\prime}} y_{k}^{\beta} \\
& =\frac{\alpha^{\prime} \geq 1, \beta \geq 1}{\sum_{\nu} \exp \left\{y_{\nu}\right\}} \\
& \leq 6 L \mathcal{R}_{2} \quad \text { [due to } 0 \leq y_{j} \leq L, \frac{\alpha^{\prime}}{q+1} \leq 1 \text { ]. }
\end{aligned}
$$

Now, let $\zeta$ be the matrix obtained from $\eta$ by replacing the diagonal entries with zeros. Then

$$
-2 I \preceq \zeta \preceq 2 I
$$


and

$$
\mathcal{J}_{1}=\frac{2 \sum_{p=2}^{\infty} \frac{1}{p !} \overbrace{\sum_{\substack{\alpha+\beta+\gamma=p+1 \\ \alpha, \beta, \gamma \geq 1}} \sum_{j, k, \ell} \zeta_{j k} \zeta_{k \ell} \zeta_{\ell j} \frac{1}{p !} y_{j}^{\alpha} y_{k}^{\beta} y_{\ell}^{\gamma}}^{\Phi(\alpha, \beta, \gamma)}}{\sum_{\nu} \exp \left\{y_{\nu}\right\}} .
$$

$\Phi(\alpha, \beta, \gamma)$ clearly is symmetric in $\alpha, \beta, \gamma$, which gives the first inequality in the following chain:

$$
\begin{aligned}
& \left|\mathcal{J}_{1}\right| \leq \frac{12 \sum_{p=2}^{\infty} \frac{1}{p !} \sum_{\substack{\alpha+\beta+\gamma=p+1 \\
1 \leq \alpha \leq \beta \geq \gamma \geq 1}}\left|\sum_{j, k, \ell} \zeta_{j k} \zeta_{k \ell} \zeta_{\ell j} \frac{1}{p !} y_{j}^{\alpha} y_{k}^{\beta} y_{\ell}^{\gamma}\right|}{\sum_{\nu} \exp \left\{y_{\nu}\right\}} \\
& 12 \sum_{p=2}^{\infty} \frac{1}{p !} \sum_{\substack{\alpha+\beta+\gamma=p+1 \\
1 \leq \alpha \leq \beta \geq \gamma \geq 1}}\left|\operatorname{Tr}\left(X^{\alpha} \zeta X^{\beta} \zeta X^{\gamma} \zeta\right)\right| \\
& =\frac{{ }^{\alpha+\beta}+\gamma=p+1}{1 \leq \alpha \leq \beta \geq \gamma \geq 1} \quad \quad\left[X=\operatorname{Diag}\left\{y_{1}, y_{2}, \ldots, y_{n}\right\}\right] \\
& 12 \sum_{p=2}^{\infty} \frac{1}{p !} \sum_{\alpha+\gamma \leq \frac{2(p+1)}{3}}^{\nu}\left|\operatorname{Tr}\left(\left[X^{\alpha} \zeta X^{\frac{p+1-2 \alpha}{2}}\right]\left[X^{\frac{p+1-2 \gamma}{2}} \zeta X^{\gamma}\right] \zeta\right)\right| \\
& \leq \frac{1 \leq \alpha, \gamma<\frac{p+1}{2}}{\sum_{\nu} \exp \left\{y_{\nu}\right\}} \\
& 24 \sum_{p=2}^{\infty} \frac{1}{p !} \sum_{\alpha+\gamma \leq \frac{2(p+1)}{3}} \overbrace{\left\|X^{\alpha} \zeta X^{\frac{p+1-2 \alpha}{2}}\right\|_{F}}^{S_{\alpha}}\left\|X^{\frac{p+1-2 \gamma}{2}} \zeta X^{\gamma}\right\|_{F} \\
& \leq \frac{1 \leq \alpha, \gamma<\frac{p+1}{2}}{\sum_{\nu} \exp \left\{y_{\nu}\right\}} \quad[\text { due to }-2 I \leq \zeta \leq 2 I] \text {. }
\end{aligned}
$$

We have

$$
S_{\alpha}^{2}=\sum_{j \neq k} y_{j}^{2 \alpha} \eta_{j k}^{2} y_{k}^{p+1-2 \alpha} .
$$

Therefore

$$
\sum_{1 \leq \alpha<\frac{p+1}{2}} S_{\alpha}^{2} \leq \sum_{j \neq k} \sum_{\substack{\mu+\tau=p+1 \\ \mu, \tau \geq 1}} \eta_{j k}^{2} y_{j}^{\mu} y_{k}^{\tau}
$$

whence

$$
\begin{aligned}
\left|\mathcal{J}_{1}\right| \leq & \frac{24 \sum_{p=2}^{\infty} \frac{1}{p !} \sum_{1 \leq \alpha, \gamma<\frac{p+1}{2}} S_{\alpha} S_{\gamma}}{\sum_{\nu} \exp \left\{y_{\nu}\right\}}=\frac{24 \sum_{p=2}^{\infty} \frac{1}{p !}\left(\sum_{1 \leq \alpha<\frac{p+1}{2}} S_{\alpha}\right)^{2}}{\sum_{\nu} \exp \left\{y_{\nu}\right\}} \leq \frac{24 \sum_{p=2}^{\infty} \frac{p}{p !} \sum_{1 \leq \alpha<\frac{p+1}{2}} S_{\alpha}^{2}}{\sum_{\nu} \exp \left\{y_{\nu}\right\}} \\
\leq & \frac{24 \sum_{q=1}^{\infty} \frac{1}{q !} \sum_{\substack{p \neq k \\
j \neq+\tau=q+2}} \sum_{j, k}^{2} y_{j}^{\mu} y_{k}^{\tau}}{\sum_{\nu} \exp \left\{y_{\nu}\right\}} .
\end{aligned}
$$

Since $0<y_{j} \leq L$, we clearly have

$$
\sum_{q=1}^{\infty} \frac{1}{q !} \sum_{j \neq k} \sum_{\substack{\mu+\tau=q+2 \\ \mu, \tau \geq 1}} \eta_{j k}^{2} y_{j}^{\mu} y_{k}^{\tau} \leq 2 L \sum_{q=1}^{\infty} \frac{1}{q !} \sum_{j \neq k} \sum_{\substack{\alpha+\beta=q+1 \\ \alpha, \beta \geq 1}} \eta_{j k}^{2} y_{j}^{\alpha} y_{k}^{\beta}
$$

and we arrive at

$$
\left|\mathcal{J}_{1}\right| \leq 48 L \mathcal{R}_{2}
$$


Combining (37), (38) and (40), we arrive at the relation

$$
\left|d^{3} f\right| \leq O(1) L d^{2} f,
$$

as claimed. - 